\title{
On Gauss-Bonnet gravity and boundary conditions in Lorentzian path-integral quantization
}

\author{
Gaurav Narain \\ Center for Gravitational Physics, Department of Space Science, Beihang University, \\ Beijing 100191, China \\ E-mail: gaunarain@gmail.com
}

ABSTRACT: Recently there has been a surge of interest in studying Lorentzian quantum cosmology using Picard-Lefschetz methods. The present paper aims to explore the Lorentzian path-integral of Gauss-Bonnet gravity in four spacetime dimensions with metric as the field variable. We employ mini-superspace approximation and study the variational problem exploring different boundary conditions. It is seen that for mixed boundary conditions non-trivial effects arise from Gauss-Bonnet sector of gravity leading to additional saddle points for lapse in some case. As an application of this we consider the No-boundary proposal of the Universe with two different settings of boundary conditions, and compute the transition amplitude using Picard-Lefschetz formalism. In first case the transition amplitude is a superposition of a Lorentzian and a Euclidean geometrical configuration leading to interference incorporating non-perturbative effects coming from Gauss-Bonnet sector of gravity. In the second case involving complex initial momentum we note that the transition amplitude is an analogue of Hartle-Hawking wave-function with non-perturbative correction coming from Gauss-Bonnet sector of gravity.

KEYwords: Models of Quantum Gravity, Nonperturbative Effects, Space-Time Symmetries, Cosmology of Theories beyond the SM

ARXIV EPRINT: 2101.04644 


\section{Contents}

1 Introduction 1

$\begin{array}{lrr}2 & \text { Mini-superspace action } & 6\end{array}$

$\begin{array}{lll}3 & \text { Boundary action and boundary conditions } & \mathbf{7}\end{array}$

3.1 Neumann boundary condition (NBC) 8

3.2 Dirichlet boundary condition (DBC) 8

$\begin{array}{lll}3.3 & \text { Mixed boundary condition (MBC) } & 10\end{array}$

4 Transition probability 11

$\begin{array}{lll}4.1 & \text { Saddle points } & 13\end{array}$

$5 \quad N_{c}$-integration via Picard-Lefschetz $\quad 15$

$\begin{array}{lll}5.1 & \text { Flow equations } & 16\end{array}$

$\begin{array}{ll}5.2 \text { Choice of contour } & 17\end{array}$

$\begin{array}{lll}5.3 \text { Flow directions } & 18\end{array}$

$\begin{array}{lll}5.4 \text { Saddle-point approximation } & 19\end{array}$

$\begin{array}{lll}6 & \text { No-boundary Universe } & 19\end{array}$

7 Complex initial momentum $\quad 25$

8 Conclusion $\quad 28$

\section{Introduction}

General relativity (GR) is the first theory to unite gravity with relativity using the notion of curved spacetime. It has been hugely successful at offering explanations for a variety of physical phenomenon ranging from astrophysical to cosmological scales. Despite its huge successes as a classical theory, the theory suffers from lack of completion at small scales as also is demonstrated by singularity theorems. When the successful framework of quantum field theory (QFT) is applied to GR, then the QFT of GR is seen to be nonrenormalizable and the resulting theory lacks predictivity [1-7]. These results although perturbative indicate the lack of compatibility of the two theories at small scales thereby suggesting that either GR or QFT or both should suitably be modified at short distances to have a well-defined ultraviolet complete theory of gravity.

Experimental observations at large distances fail to agree with the predictions of GR coupled with standard model. This although doesn't imply failure of GR, but it does hint that the coupled system of standard model and GR is missing something. Over the years 
researchers have tried to address this issue in different ways: (1) modify the standard model by incorporating dark-matter and dark-energy while keeping GR to be unmodified, (2) modify gravitational dynamics at large distances keeping the standard model unmodified or (3) modify both GR and standard model. In the case (2) and (3) one goes beyond GR.

Numerous model has been proposed over the years to explain such deviations at both ends of energy scales. For example at ultra short length scales, motivated by lack of renormalizabilty of GR (which has only two time derivatives of the metric field) proposals have been made to incorporate higher-time derivatives of the metric field. Such modifications of GR are collectively referred to as higher-derivative theories of gravity. It has been noticed that incorporating higher-derivatives although addresses issues of renormalizability in four spacetime dimensions but the theory lacks unitarity [8-10]. Some efforts have been made to tackle this unwanted problem in the perturbative framework [11-14], in asymptotic safety approach [15, 16] and 'Agravity' [17]. However, one also notices an important fact that equation of motion in such UV modified theories have higher-time derivatives (more than two) of metric field. One wonders at the presence of such higher-time derivatives which although addresses renormalizability but also are responsible for lack of tree-level unitarity in theory.

Overtime a need arose of having a modification of GR which consist of higherderivatives of the metric field, but when contributions from all such terms are summed over then the highest order of time derivative is only two. The Gauss-Bonnet (GB) gravity in four spacetime dimensions is one such simple modification of the GR, which satisfies exactly this requirement. Here the dynamical evolution equations of field remains unaffected. In four spacetime dimension GB sector of gravity action is also topological and doesn't play any role in dynamical evolution of spacetime metric. However, they play a key role in path-integral quantization of gravity where it is used to classify topologies and has an important role to play at boundaries. The bulk Gauss-Bonnet gravity action is following

$$
S=\frac{1}{16 \pi G} \int \mathrm{d}^{D} x \sqrt{-g}\left[-2 \Lambda+R+\alpha\left(R_{\mu \nu \rho \sigma} R^{\mu \nu \rho \sigma}-4 R_{\mu \nu} R^{\mu \nu}+R^{2}\right)\right],
$$

where $G$ is the Newton's gravitational constant, $\Lambda$ is the cosmological constant term, $\alpha$ is the Gauss-Bonnet coupling and $D$ is spacetime dimensionality. The mass dimensions of various couplings are: $[G]=M^{2-D},[\Lambda]=M^{2}$ and $[\alpha]=M^{-2}$. This action falls in the class of Lovelock-Lanczos gravity theories [18-20], and are a special class of higher-derivative gravity where equation of motion for the metric field remains second order in time.

Motivated by the above properties of GB gravity, it is worth asking how the pathintegral of the metric field behaves when its gravitational action is given by eq. (1.1)? My interest in this paper is to study such a path-integral in a slightly simpler setting: the context of quantum cosmology where the issues regarding various kind of boundary conditions are easier to investigate [21-25]. We start by considering a generic metric respecting spatial homogeneity and isotropicity in $D$ spacetime dimensions. It is the FLRW metric in arbitrary spacetime dimension with dimensionality $D$. In polar co-ordinates 
$\left\{t_{p}, r, \theta, \cdots\right\}$ the FLRW metric can be expressed as

$$
\mathrm{d} s^{2}=-N_{p}^{2}\left(t_{p}\right) \mathrm{d} t_{p}^{2}+a^{2}\left(t_{p}\right)\left[\frac{\mathrm{d} r^{2}}{1-k r^{2}}+r^{2} \mathrm{~d} \Omega_{D-2}^{2}\right] .
$$

It consists of two unknown time-dependent functions: lapse $N_{p}\left(t_{p}\right)$ and scale-factor $a\left(t_{p}\right)$. Here $k=(0, \pm 1)$ is the curvature, and $\mathrm{d} \Omega_{D-2}$ is the metric corresponding to unit sphere in $D-2$ spatial dimensions. This is the mini-superspace approximation of the metric. This is a gross simplification of the original gravitational theory in a sense as we no longer have gravitational waves. However, we do retain the diffeomorphism invariance of the time co-ordinate $t_{p}$ and the dynamical scale-factor $a\left(t_{p}\right)$. This simple setting is enough to investigate the effects the GB-modification of GR in the gravitational path-integral.

The Feynman path-integral for the reduced theory can be written as

$$
G\left[\mathrm{bd}_{0}, \mathrm{bd}_{1}\right]=\int_{\mathrm{bd}_{0}}^{\mathrm{bd}_{1}} \mathcal{D} N_{p} \mathcal{D} \pi \mathcal{D} a\left(t_{p}\right) \mathcal{D} p \mathcal{D C} \mathcal{D} \bar{P} \exp \left[\frac{i}{\hbar} \int_{0}^{1} \mathrm{~d} t_{p}\left(N_{p}^{\prime} \pi+a^{\prime} p+\mathcal{C}^{\prime} \bar{P}-N_{p} H\right)\right],
$$

where beside the scale-factor $a\left(t_{p}\right)$, lapse $N_{p}$ and fermionic ghost $\mathcal{C}$ we also have their corresponding conjugate momenta given by $p, \pi$ and $\bar{P}$ respectively. Here $\left({ }^{\prime}\right)$ denotes derivative with respect to $t_{p}$. The original path-integral measure becomes a measure over all the variables. The time $t_{p}$ co-ordinate can be chosen to range from $0 \leq t_{p} \leq 1$ without compromising on generality. Here $\mathrm{bd}_{0}$ and $\mathrm{bd}_{1}$ refers to field configuration at initial $\left(t_{p}=0\right)$ and final $\left(t_{p}=1\right)$ boundary respectively. The Hamiltonian constraint $H$ consists of two parts

$$
H=H_{G B}[a, p]+H_{\mathrm{gh}}[N, \pi, \mathcal{C}, \bar{P}],
$$

the Hamiltonian corresponding to Gauss-Bonnet gravity action is denoted by $H_{G B}$ and the Batalin-Fradkin-Vilkovisky (BFV) [26] ghost Hamiltonian is denoted by $H_{\text {gh }} \cdot{ }^{1}$ The minisuperspace approximation still retains diffeomorphism invariance which show up as a time reparametrization symmetry. This invariance can be broken by fixing the proper-time gauge $N_{p}^{\prime}=0$. For more elaborate discussion on BFV quantization process and ghost see [27-29].

In the mini-superspace approximation most of the path-integral in eq. (1.3) can be performed analytically leaving behind the following path-integral

$$
G\left[\mathrm{bd}_{0}, \mathrm{bd}_{1}\right]=\int_{0^{+}}^{\infty} \mathrm{d} N_{p} \int_{\mathrm{bd}_{0}}^{\mathrm{bd}_{1}} \mathcal{D} a\left(t_{p}\right) e^{i S\left[a, N_{p}\right] / \hbar} .
$$

This residual path-integral is easy to interpret. The path-integral $\int \mathcal{D} a\left(t_{p}\right) e^{i S\left[a, N_{p}\right] / \hbar}$ represent the quantum-mechanical transitional amplitude for the Universe to evolve from one configuration to another in proper time $N_{p}$. The integration over lapse-function $N_{p}$

\footnotetext{
${ }^{1}$ The BFV ghost is an extension of the usual Fadeev-Popov ghost which is based on BRST symmetry. In usual gauge theories the constraint algebra forms a Lie algebra, while the constraint algebra doesn't closes in case of diffeomorphism invariant gravitational theories. For this reason one needs BFV quantization process. In the case of mini-superspace approximation however, we have only one constraint $H$. In this approximation therefore the algebra trivially closes leaving the distinction between two quantization process irrelevant. Nevertheless BFV quantization is still preferable.
} 
indicates that one has to consider paths of every proper duration $0<N_{p}<\infty$. Such a choice leads to causal ordering of the two field configuration $b_{0}$ and $b_{1}$ as shown in [30], where $a_{0}<a_{1}$ will imply expanding Universe while $a_{0}>a_{1}$ will imply contracting Universe. In this paper we are interested in studying this residual path-integral for the case of Gauss-Bonnet gravity where we expect that the choice of boundary configurations may or may-not give rise to non-trivial features coming from the Gauss-Bonnet term in the gravitational action.

Ones task is now reduced to the study of functional integral in eq. (1.5). In general the standard methodology to deal with flat spacetime Lorentzian functional integrals of non-gravitational QFT is to Wick rotate the Lorentzian time co-ordinate and go to Euclideanised time, which for example in the current case will mean $t_{p} \rightarrow i \tau_{p}$, where $\tau_{p}$ is the Euclideanised time. Such a rotation of time co-ordinate analytically transforms the flat spacetime Lorentzian path-integral to a Euclidean path-integral with an exponentially suppressed weight factor, which is convergent and well-defined.

In case of gravity the situation is not so straightforward. One can in principle aim to directly study the following Euclidean gravitational path-integral

$$
G\left[\mathrm{bd}_{0}, \mathrm{bd}_{1}\right]=\int_{\mathrm{bd}_{0}}^{\mathrm{bd}_{1}} \mathcal{D} g_{\mu \nu} \exp \left(-I\left[g_{\mu \nu}\right]\right) .
$$

Here $g_{\mu \nu}$ is the metric whose corresponding Euclidean action $I\left[g_{\mu \nu}\right]$ appears in the exponential. The motivation to study directly such an Euclidean gravitational path-integral stems from fact that similar but non-gravitational Euclidean path-integral arises in flat spacetime QFT which are obtained by analytic continuation of a meaningful time co-ordinate. In case of gravity one is skeptical about the relation between the Euclidean and the corresponding Lorentzian path-integral, in a sense whether the two can be analytically related in some way. This is because it is not always possible to have a meaningful time coordinate in generic curved spacetime. Moreover, the Euclidean gravitational path-integral given in eq. (1.6) suffers from the conformal factor problem, where the path-integral over the scale-factor is unbounded from below [31]. This implies that the Euclidean gravitational path-integral is not convergent and is ill-defined. This is unlike the situation in flat spacetime non-gravitational QFT where the Euclidean path-integral of the corresponding Lorentzian path-integral is convergent and well-defined. ${ }^{2}$

\footnotetext{
${ }^{2}$ As a side remark it should be emphasised that flat spacetime has a meaningful time co-ordinate and enjoys the properties of global symmetries to cast the Lorentz group in to a compact rotation group under a transformation of the time co-ordinate. Such a beauty is not present in a generic curved spacetime. This implies that the standard methodology of Wick-rotation used for defining sensible quantum field theory (QFT) on flat spacetime is difficult to generalise reliably in a generic curved spacetime where 'time' is just a parameter. The Feynman $+i \epsilon$-prescription in flat spacetime QFT is a systematic way to choose a convergent integration contour for an otherwise highly oscillatory integral. It naturally implements causality in path-integral in a systematic manner by requiring that the Euclideanised version of two-point function must satisfy Osterwalder-Schrader positivity. Such benefits exist only in flat spacetime and don't get automatically inherited to generic Lorentzian curved spacetime. The situation gets even more cumbersome when spacetime becomes dynamical due to gravity and/or gravitational field is also quantized. Some attempts to incorporate Wick-rotation sensibly in curved spacetime have been made in [32-35]. However, more work needs to be done for it to mature.
} 
Picard-Lefschetz theory offers a systematic methodology to carefully handle oscillatory path-integrals like the one in eq. (1.5). It is an extension of the Wick-rotation prescription to define a convergent functional integral on a generic curved spacetime. In this framework one uniquely finds contours in the complexified plane along which the integrand is well-behaved. By definition the oscillatory integral along the original integration contour becomes wellbehaved and non-oscillatory along the new contour. Such contour-lines are termed Lefschetz thimbles. This framework is based on complex analysis has been recently used in context of Lorentzian quantum cosmology [38-40], where the authors studied gravitational pathintegral in the mini-superspace approximation for Einstein-Hilbert gravity. ${ }^{3}$

Once it is possible to have a well-defined convergent Lorentzian path-integral using Picard-Lefschetz theory, one can then explore the various choices of allowed boundary conditions. In the context of Euclidean quantum gravity, whose path-integral suffers from conformal factor problem [31, 45], it was realised that a sensible choice of initial conditions and integration contour leads to a well-defined convergent path-integral [46-48]. This has motivated people to follow the same footsteps to study boundary condition choices in the context of Lorentzian gravitational path-integral which become well-defined using the framework of Picard-Lefschetz [38-40].

Motivated by these ideas we set to investigate the gravitational path-integral for the Gauss-Bonnet gravity in the mini-superspace approximation using the technology of PicardLefschetz theory. We start by varying the action with respect to field and study the nature of surface terms. We explore three different choice of boundary conditions: Dirichlet (D), Neumann $(\mathrm{N})$ and Mixed (M) boundary conditions (BC). In each case the surface-terms are either zero or contribute same as in GR, except in the case of mixed-boundary conditions (MBC) where the surface terms gets an additional non-trivial contribution from Gauss-Bonnet sector of gravitational action. This has interesting consequences in the followup study of the path-integral. Mixed boundary conditions (MBC) are interesting and have also been previously explored in the context of Einstein-Hilbert gravity $[49,50]$ in relation with no-boundary proposal of the Universe. We explore MBC in the context of gravitational path-integral of Gauss-Bonnet gravity, and find some non-trivial contribution coming from Gauss-Bonnet sector. As a special case we consider the no-boundary proposal of the Universe and find interesting features arising from the Gauss-Bonnet sector of gravity action.

The outline of paper is follows: in section 1 we motivates our interest in studying this problem. In section 2 we discuss the mini-superspace approximation and compute the mini-superspace action of theory. In section 3 we discuss the action variation and study the various boundary conditions. In section 4 we consider the path-integral of gravity in mini-superspace approximation and start to compute the transition probability in saddle point approximation. Section 5 studies the integration over lapse in complex space via Picard-Lefschetz. In section 6 we study the no-boundary proposal of Universe with

\footnotetext{
${ }^{3}$ It should be mentioned that usage of complex analysis was also made in past to study Euclidean gravitational path-integrals in eq. (1.6) which are know to suffer from conformal factor problem [36, 37]. In the context of Euclidean quantum cosmology usage of complex analysis was made to explore issues regarding initial conditions: tunnelling proposal [41-43] and no-boundary proposal [36, 37, 44].
} 
mixed boundary conditions. In section 7 we analyse the Hartle-Hawking wave-function using Lorentzian path-integral and the corrections it receive due to Gauss-Bonnet sector of gravity. We finish off by presenting a conclusion and outlook in section 8 .

\section{Mini-superspace action}

The FLRW metric given in eq. (1.2) is conformally related to flat metric and hence its Weyl-tensor $C_{\mu \nu \rho \sigma}=0$. The non-zero entries of the Riemann tensor are [51-53]

$$
\begin{aligned}
& R_{0 i 0 j}=-\left(\frac{a^{\prime \prime}}{a}-\frac{a^{\prime} N_{p}^{\prime}}{a N_{p}}\right) g_{i j}, \\
& R_{i j k l}=\left(\frac{k}{a^{2}}+\frac{a^{\prime 2}}{N_{p}^{2} a^{2}}\right)\left(g_{i k} g_{j l}-g_{i l} g_{j k}\right),
\end{aligned}
$$

where $g_{i j}$ is the spatial part of the FLRW metric and $\left({ }^{\prime}\right)$ denotes derivative with respect to $t_{p}$. For the Ricci-tensor the non-zero components are

$$
\begin{aligned}
& R_{00}=-(D-1)\left(\frac{a^{\prime \prime}}{a}-\frac{a^{\prime} N_{p}^{\prime}}{a N_{p}}\right) \\
& R_{i j}=\left[\frac{(D-2)\left(k N_{p}^{2}+a^{\prime 2}\right)}{N_{p}^{2} a^{2}}+\frac{a^{\prime \prime} N_{p}-a^{\prime} N_{p}^{\prime}}{a N_{p}^{3}}\right] g_{i j},
\end{aligned}
$$

while the Ricci-scalar for FLRW is given by

$$
R=2(D-1)\left[\frac{a^{\prime \prime} N_{p}-a^{\prime} N_{p}^{\prime}}{a N_{p}^{3}}+\frac{(D-2)\left(k N_{p}^{2}+a^{\prime 2}\right)}{2 N_{p}^{2} a^{2}}\right] .
$$

In the case of Weyl-flat metrics one can express Riemann tensor in terms of Ricci-tensor and Ricci scalar.

$$
R_{\mu \nu \rho \sigma}=\frac{R_{\mu \rho} g_{\nu \sigma}-R_{\mu \sigma} g_{\nu \rho}+R_{\nu \sigma} g_{\mu \rho}-R_{\nu \rho} g_{\mu \sigma}}{D-2}-\frac{R\left(g_{\mu \rho} g_{\nu \sigma}-g_{\mu \sigma} g_{\nu \rho}\right)}{(D-1)(D-2)} .
$$

Due to this identity we have

$$
R_{\mu \nu \rho \sigma} R^{\mu \nu \rho \sigma}=\frac{4}{D-2} R_{\mu \nu} R^{\mu \nu}-\frac{2 R^{2}}{(D-1)(D-2)}
$$

It allow us to simplify our Gauss-Bonnet gravity action for Weyl-flat metrics.

$$
\begin{gathered}
\int \mathrm{d}^{D} x \sqrt{-g}\left(R_{\mu \nu \rho \sigma} R^{\mu \nu \rho \sigma}-4 R_{\mu \nu} R^{\mu \nu}+R^{2}\right) \\
=\frac{D-3}{D-2} \int \mathrm{d}^{D} x \sqrt{-g}\left(-R_{\mu \nu} R^{\mu \nu}+\frac{D R^{2}}{D-1}\right) .
\end{gathered}
$$


On plugging the FLRW metric of eq. (1.2) in the action in eq. (1.1) we get an action for $a\left(t_{p}\right)$ and $N_{p}\left(t_{p}\right)$.

$$
\begin{aligned}
S= & \frac{V_{D-1}}{16 \pi G} \int \mathrm{d} t_{p}\left[\frac { a ^ { D - 3 } } { N _ { p } ^ { 2 } } \left\{(D-1)(D-2) k N_{p}^{3}-2 \Lambda a^{2} N_{p}^{3}-2(D-1) a a^{\prime} N_{p}^{\prime}\right.\right. \\
& \left.+(D-1)(D-2) a^{\prime 2} N_{p}+2(D-1) N_{p} a a^{\prime \prime}\right\}+(D-1)(D-2)(D-3) \alpha\left\{\frac{a^{D-5}(D-4)}{N_{p}^{3}}\right. \\
& \left.\left.\times\left(k N_{p}^{2}+a^{\prime 2}\right)^{2}+4 a^{D-4} \frac{\mathrm{d}}{\mathrm{d} t_{p}}\left(\frac{k a^{\prime}}{N_{p}}+\frac{a^{\prime 3}}{3 N_{p}^{3}}\right)\right\}\right],
\end{aligned}
$$

where $V_{D-1}$ is the volume of $D-1$ dimensional space and is given by,

$$
V_{D-1}=\frac{\Gamma(1 / 2)}{\Gamma(D / 2)}\left(\frac{\pi}{k}\right)^{(D-1) / 2} .
$$

In $D=4$ we notice that in the GB-sector terms proportional $\alpha$ either vanish or are total time-derivatives. The mini-superspace gravitational action becomes following in $D=4$

$S=\frac{V_{3}}{16 \pi G} \int \mathrm{d} t_{p}\left[6 k a N_{p}-2 \Lambda a^{3} N_{p}-\frac{6 a^{2} a^{\prime} N_{p}^{\prime}}{N_{p}}+\frac{6 a a^{\prime 2}}{N_{p}}+\frac{6 a^{\prime \prime} a^{2}}{N_{p}}+24 \alpha \frac{\mathrm{d}}{\mathrm{d} t_{p}}\left(\frac{k a^{\prime}}{N_{p}}+\frac{a^{\prime 3}}{3 N_{p}^{3}}\right)\right]$,

This action can be recast in to a more appealing form by a rescaling of lapse and scale factor.

$$
N_{p}\left(t_{p}\right) \mathrm{d} t_{p}=\frac{N(t)}{a(t)} \mathrm{d} t, \quad q(t)=a^{2}(t) .
$$

This set of transformation changes our original metric in eq. (1.2) into following

$$
\mathrm{d} s^{2}=-\frac{N^{2}}{q(t)} \mathrm{d} t^{2}+q(t)\left[\frac{\mathrm{d} r^{2}}{1-k r^{2}}+r^{2} \mathrm{~d} \Omega_{D-2}^{2}\right],
$$

and our action in $D=4$ given in eq. (2.9) changes to following simple form

$$
S=\frac{V_{3}}{16 \pi G} \int \mathrm{d} t\left[(6 k-2 \Lambda q) N+\frac{3 \dot{q}^{2}}{2 N}+3 q \frac{\mathrm{d}}{\mathrm{d} t}\left(\frac{\dot{q}}{N}\right)+24 \alpha \frac{\mathrm{d}}{\mathrm{d} t}\left(\frac{k \dot{q}}{2 N}+\frac{\dot{q}^{3}}{24 N^{3}}\right)\right] .
$$

Here $\left({ }^{\circ}\right)$ here represent derivative with respect to time $t$. It is worth noting the GB-part of action appears as a total derivative term. It will later be seen that this part plays a crucial role in the action for the lapse $N$ and will result in additional saddle points. In the path-integral this term will play crucial role as it will in some sense be incorporating topological corrections.

\section{Boundary action and boundary conditions}

To find the boundary action and the relevant set of boundary conditions we start by varying the action in eq. (2.12) with respect to $q(t)$. From now on we work in the ADM gauge $\dot{N}=0$, which implies that $N(t)=N_{c}$ (constant). We write

$$
q(t)=\bar{q}(t)+\epsilon \delta q(t)
$$


where $\bar{q}(t)$ satisfy the equation of motion, $\delta q(t)$ is the fluctuation around it and $\epsilon$ is parameter used to keep a track of the order of fluctuation terms. Plugging this in eq. (2.12) and expanding to first order in $\epsilon$ we have

$$
\delta S=\frac{\epsilon V_{3}}{16 \pi G} \int_{0}^{1} \mathrm{~d} t\left[\left(-2 \Lambda N_{c}+\frac{3 \ddot{q}}{N_{c}}\right) \delta q+\frac{3}{N_{c}} \frac{\mathrm{d}}{\mathrm{d} t}(q \delta \dot{q})+24 \alpha \frac{\mathrm{d}}{\mathrm{d} t}\left\{\left(\frac{k}{2 N_{c}}+\frac{\dot{q}^{2}}{8 N_{c}^{3}}\right) \delta \dot{q}\right\}\right] .
$$

There will also be second order terms, but for the purpose of having a sensible boundary value problem for the equation of motion this is sufficient. We notice that there are two total time-derivative pieces in the above equation which will be responsible for fixing appropriate boundary conditions. The term proportional to $\delta q$ gives the equation of motion for $q$

$$
\ddot{q}=\frac{2}{3} \Lambda N_{c}^{2} .
$$

This is easy to solve and its general solution is

$$
q(t)=\frac{\Lambda N_{c}^{2}}{3} t^{2}+c_{1} t+c_{2}
$$

where $c_{1,2}$ are constants and will be determined based on the boundary conditions. The total-derivative terms in the above will result in a collection of boundary terms

$$
S_{\text {bdy }}=\frac{\epsilon V_{3}}{16 \pi G}\left[\frac{3}{N_{c}}\left(q_{1} \delta \dot{q}_{1}-q_{0} \delta \dot{q}_{0}\right)+24 \alpha\left\{\left(\frac{k \delta \dot{q}_{1}}{2 N_{c}}+\frac{\dot{q}_{1}^{2} \delta \dot{q}_{1}}{8 N_{c}^{3}}\right)-\left(\frac{k \delta \dot{q}_{0}}{2 N_{c}}+\frac{\dot{q}_{0}^{2} \delta \dot{q}_{0}}{8 N_{c}^{3}}\right)\right\}\right]
$$

where

$$
q_{0}=q(t=0), \quad q_{1}=q(t=1), \quad \dot{q}_{0}=\dot{q}(t=0), \quad \dot{q}_{1}=\dot{q}(t=1) .
$$

\subsection{Neumann boundary condition (NBC)}

If we impose Neumann boundary condition (NBC) which is fixing $\dot{q}$ at both the ends of the $q$-trajectory $[23,49]$. Then we notice that the surface term in eq. (3.5) vanish completely.

$$
\left.\dot{q}_{0,1}\right|_{\mathrm{NBC}}=\text { fixed }\left.\Rightarrow \delta \dot{q}_{0,1}\right|_{\mathrm{NBC}}=0
$$

where the $\left.\right|_{\text {NBC }}$ refers to imposing Neumann boundary condition. However, it is soon realised that with this boundary condition the constant $c_{1,2}$ appearing in the solution to equation of motion (3.4) cannot be fixed uniquely. In particular $c_{2}$ is left undetermined while $c_{1}$ will have two different values. This implies that it is not a well-posed problem as it leads to inconsistencies. This boundary condition cannot be imposed even though the surface term in eq. (3.5) vanishes entirely and one doesn't have to incorporate any additional boundary action.

\subsection{Dirichlet boundary condition (DBC)}

In this boundary condition we fix the value of $q$ at the two end points. This means we have

$$
\left.q_{0,1}\right|_{\mathrm{DBC}}=\text { fixed }\left.\Rightarrow \delta q_{0,1}\right|_{\mathrm{DBC}}=0
$$


where the $\left.\right|_{\text {DBC }}$ refers to imposing Dirichlet boundary condition. Our surface contribution in eq. (3.5) doesn't vanish under the imposition of this boundary condition. In the case when $\alpha=0$ (only Einstein-Hilbert gravity), then in order to have a sensible Dirichlet boundary value problem one has to add an extra boundary action. This is the well known Gibbon-Hawking-York term [21, 22, 31], which in mini-superspace reduces to

$$
S_{\mathrm{GHY}}=-\left.\frac{V_{3}}{16 \pi G} \frac{3 q \dot{q}}{N_{c}}\right|_{0} ^{1}=-\frac{V_{3}}{16 \pi G}\left(\frac{3 q_{1} \dot{q}_{1}}{N_{c}}-\frac{3 q_{0} \dot{q}_{0}}{N_{c}}\right) .
$$

On varying the $S_{\mathrm{GHY}}$ action it is noticed that it cancels the $\delta \dot{q}$ terms at the boundary in eq. (3.5) for $\alpha=0$. It therefore sets up a successful imposition dirichlet boundary condition, atleast for the Einstein-Hilbert gravity part of theory, thereby leading to a consistent solution to equation of motion.

But the same thing can not be implemented for the Gauss-Bonnet sector of gravitational surface terms. They will be proportional to $f(\dot{q}) \delta \dot{q}$, where $f(\dot{q})=\left(1 / 2 N_{c}+\dot{q}^{2} / 8 N_{c}^{3}\right)$. In principle one can construct a possible surface term for the Gauss-Bonnet sector.

$$
\left.S_{G B}\right|_{\mathrm{bdy}}=\left.F(q, \dot{q})\right|_{0} ^{1} .
$$

During the process variation of action with respect to $q$ to compute equation of motion, this surface term on variation will lead to

$$
\left.\epsilon\left(\frac{\partial F}{\partial q} \delta q+\frac{\partial F}{\partial \dot{q}} \delta \dot{q}\right)\right|_{0} ^{1}
$$

Then in order to cancel the surface contribution proportional to $\alpha$ in eq. (3.5), we notice that implies

$$
\begin{gathered}
\left.\epsilon \frac{\partial F}{\partial \dot{q}} \delta \dot{q}\right|_{0} ^{1}+\left.\frac{\epsilon V_{3}}{16 \pi G} 24 \alpha\left\{\left(\frac{k}{2 N_{c}}+\frac{\dot{q}^{2}}{8 N_{c}^{3}}\right) \delta \dot{q}\right\}\right|_{0} ^{1}=0, \\
\Rightarrow \quad F(q, \dot{q})=-\frac{V_{3}}{16 \pi G} 24 \alpha\left(\frac{k \dot{q}}{2 N_{c}}+\frac{\dot{q}^{3}}{24 N_{c}^{3}}\right)+g(q) .
\end{gathered}
$$

As the Gauss-Bonnet surface part in eq. (3.5) doesn't have any term proportional to $\delta q_{0}$ or $\delta q_{1}$, so this implies that $g^{\prime}(q)=0$, which can be fixed to zero. Then the total boundary action is a summation of Gibbon-Hawking term from eq. (3.9) and Gauss-Bonnet part coming from eq. (3.12).

$$
S_{\text {surface }}=S_{\mathrm{GHY}}-\frac{V_{3}}{16 \pi G} 24 \alpha\left(\frac{k \dot{q}}{2 N_{c}}+\frac{\dot{q}^{3}}{24 N_{c}^{3}}\right) .
$$

This when added to the boundary contributions coming from varying the bulk action results in complete cancelation of the terms proportional to $\alpha$. As a result it doesn't lead to any non-trivial contributions coming from Gauss-Bonnet sector. However, for Dirichlet boundary conditions the equation of motion can still be solved without any inconsistencies, but the gravitational path-integral will not have non-trivial features coming from GaussBonnet sector of gravitational action. In a sense if our motivation is look for situations where Gauss-Bonnet piece of gravitational action contribute non-trivially then DBC doesn't fall in the category. 


\subsection{Mixed boundary condition (MBC)}

After not being able to have a consistent boundary value problem with Neumann boundary conditions and lack of obtaining non-trivial effects in the case of Dirichlet boundary conditions, we next consider the situation with mixed boundary conditions where we specify $q$ at one end and $\dot{q}$ at another end. Similar mixed boundary conditions have also been investigated in $[25,49,50,54]$, here inspired by their work we consider applying them in case of Gauss-Bonnet gravity.

In this case there are two possibilities:

$$
\begin{aligned}
& \text { Case (a) : Specify } q_{0} \text { and } \dot{q}_{1} \Rightarrow \delta q_{0}=\delta \dot{q}_{1}=0, \\
& \text { Case (b) : Specify } \dot{q}_{0} \text { and } q_{1} \Rightarrow \delta q_{1}=\delta \dot{q}_{0}=0 .
\end{aligned}
$$

We will consider each of this cases individually in more detail later in paper. But first we study the boundary action that is needed for each of these. The surface action for each of these is

$$
\begin{aligned}
& S_{\text {surface }}^{(a)}=\frac{V_{3}}{16 \pi G}\left[\frac{3 q_{0} \dot{q}_{0}}{N_{c}}+24 \alpha\left(\frac{k \dot{q}_{0}}{2 N_{c}}+\frac{\dot{q}_{0}^{3}}{24 N_{c}^{3}}\right)\right], \\
& S_{\text {surface }}^{(b)}=-\frac{V_{3}}{16 \pi G}\left[\frac{3 q_{1} \dot{q}_{1}}{N_{c}}+24 \alpha\left(\frac{k \dot{q}_{1}}{2 N_{c}}+\frac{\dot{q}_{1}^{3}}{24 N_{c}^{3}}\right)\right] .
\end{aligned}
$$

During the computation of equation of motion, each of them can be varied and added to the boundary action in eq. (3.5). This will result in

$$
\begin{aligned}
& S_{\mathrm{bdy}}+\delta S_{\text {surface }}^{(a)}=\frac{\epsilon V_{3}}{16 \pi G}\left[\frac{3}{N_{c}}\left(q_{1} \delta \dot{q}_{1}+\dot{q}_{0} \delta q_{0}\right)+24 \alpha\left(\frac{k \delta \dot{q}_{1}}{2 N_{c}}+\frac{\dot{q}_{1}^{2} \delta \dot{q}_{1}}{8 N_{c}^{3}}\right)\right], \\
& S_{\text {bdy }}+\delta S_{\text {surface }}^{(b)}=-\frac{\epsilon V_{3}}{16 \pi G}\left[\frac{3}{N_{c}}\left(\dot{q}_{1} \delta q_{1}+\dot{q}_{0} \delta q_{0}\right)+24 \alpha\left(\frac{k \delta \dot{q}_{0}}{2 N_{c}}+\frac{\dot{q}_{0}^{2} \delta \dot{q}_{0}}{8 N_{c}^{3}}\right)\right] .
\end{aligned}
$$

From this one immediately notices that in former case $(a)$ if we fix $q_{0}$ and $\dot{q}_{1}$ then r.h.s. of eq. (3.17) vanishes. Similarly in the later case $(b)$ if we fix $q_{1}$ and $\dot{q}_{0}$ then the r.h.s. of the eq. (3.18) vanishes. In this way the boundary value problem is well-posed. Moreover the total action of theory is

$$
\begin{aligned}
S_{\text {tot }}^{(a)}=S+S_{\text {surface }}^{(a)}= & \frac{V_{3}}{16 \pi G} \int \mathrm{d} t\left[(6 k-2 \Lambda q) N_{c}+\frac{3 \dot{q}^{2}}{2 N_{c}}+\frac{3 q \ddot{q}}{N_{c}}\right] \\
& +\frac{V_{3}}{16 \pi G}\left[\frac{3 q_{0} \dot{q}_{0}}{N_{c}}+24 \alpha\left(\frac{k \dot{q}_{1}}{2 N_{c}}+\frac{\dot{q}_{1}^{3}}{24 N_{c}^{3}}\right)\right], \\
S_{\text {tot }}^{(b)}=S+S_{\text {surface }}^{(b)}= & \frac{V_{3}}{16 \pi G} \int \mathrm{d} t\left[(6 k-2 \Lambda q) N_{c}+\frac{3 \dot{q}^{2}}{2 N_{c}}+\frac{3 q \ddot{q}}{N_{c}}\right] \\
& -\frac{V_{3}}{16 \pi G}\left[\frac{3 q_{1} \dot{q}_{1}}{N_{c}}+24 \alpha\left(\frac{k \dot{q}_{0}}{2 N_{c}}+\frac{\dot{q}_{0}^{3}}{24 N_{c}^{3}}\right)\right] .
\end{aligned}
$$


In each of these cases one can compute the momentum corresponding to field variable $q(t)$ by varying the bulk Lagrangian with respect to $\dot{q}$. This is given by

$$
\pi=\frac{\delta \mathcal{L}}{\delta \dot{q}}=\frac{3 \dot{q}}{N_{c}} .
$$

It should be noted the bulk momentum in both the cases is same.

The variational problem in the two cases is well-posed resulting in equation of motion whose solution can be found consistently. The solution to equation of motion in each of these cases is given by

$$
\begin{aligned}
& q^{(a)}(t)=\frac{\Lambda N_{c}^{2}}{3} t^{2}+\left(\dot{q}_{1}-\frac{2 \Lambda N_{c}^{2}}{3}\right) t+q_{0}, \\
& q^{(b)}(t)=\frac{\Lambda N_{c}^{2}}{3} t^{2}+\dot{q}_{0}(t-1)+\left(q_{1}-\frac{\Lambda N_{c}^{2}}{3}\right) .
\end{aligned}
$$

These solution can be plugged back in corresponding action of theory in eq. (3.19) and (3.20) to obtain action for the lapse $N_{c}$. The lapse action for the two cases is given by,

$$
\begin{aligned}
& S_{\text {tot }}^{(a)}=\frac{V_{3}}{16 \pi G}\left[6 k N_{c}+\frac{3 \dot{q}_{1}\left(2 q_{0}+\dot{q}_{1}\right)}{2 N_{c}}-\left(2 q_{0}+\dot{q}_{1}\right) N_{c} \Lambda+\frac{2 N_{c}^{3} \Lambda^{2}}{9}+\frac{\alpha \dot{q}_{1}}{N_{c}}\left(12 k+\frac{\dot{q}_{1}^{2}}{N_{c}^{2}}\right)\right], \\
& S_{\text {tot }}^{(b)}=\frac{V_{3}}{16 \pi G}\left[6 k N_{c}+\frac{3 \dot{q}_{0}\left(\dot{q}_{0}-2 q_{1}\right)}{2 N_{c}}+\left(\dot{q}_{0}-2 q_{1}\right) N_{c} \Lambda+\frac{2 N_{c}^{3} \Lambda^{2}}{9}-\frac{\alpha \dot{q}_{0}}{N_{c}}\left(12 k+\frac{\dot{q}_{0}^{2}}{N_{c}^{2}}\right)\right] .
\end{aligned}
$$

The lapse action include non-trivial features coming from the Gauss-Bonnet sector of gravitational action, which arise in the case of MBC. In the following we will study these two cases in more detail.

\section{Transition probability}

Generically once the action of a theory is known at the classical level then it can be used in the path-integral to study the behaviour of the corresponding quantum theory. In the case investigated in present paper the well-known classical action of Einstein-Hilbert gravity is modified by inclusion of Gauss-Bonnet gravity terms which is topological in four spacetime dimensions. Although such topological extensions doesn't affect dynamical evolution of fields at the classical level as has been noted in the previous section, but their presence play a crucial role in dictating the choice of boundary conditions.

In the case of gravitational path-integral one can study a simpler situation by restricting oneself to the mini-superspace approximation. Within this approximation one can precisely ask the following question: what is the transition amplitude from one 3-geometry to another? Is it possible to address this directly in Lorentzian signature? and what is the role played by boundary conditions in the computation of this transition amplitude given by path-integral? The relevant quantity that we are interested in can be expressed in mini-superspace approximation as follows (see $[38,46]$ for the Euclidean gravitational path-integral in mini-superspace approximation)

$$
G\left[\mathrm{bd}_{0}, \mathrm{bd}_{1}\right]=\int_{0^{+}}^{\infty} \mathrm{d} N_{c} \int_{\mathrm{bd}_{0}}^{\mathrm{bd}_{1}} \mathcal{D} q(t) \exp \left(\frac{i}{\hbar} S_{\text {tot }}\right),
$$


where $b_{0}$ and $b_{1}$ are initial and final boundary configurations respectively. The pathintegral over $q(t)$ is performed such that it respects those boundary conditions. For our present case the above path-integral will be analysed with mixed boundary conditions as discussed in eq. (3.14) in previous section. $S_{\text {tot }}$ is the total action incorporating the appropriate boundary condition as given in (3.190 and (3.20) respectively. The original contour of integration for $N_{c}$ is $\left(0^{+}, \infty\right)$. This contour integral over $N_{c}$ will be computed using the technology of Picard-Lefschetz theory.

We start by considering the fluctuations around the solution to equation of motion, which has been obtained previously respecting the boundary conditions.

$$
q(t)=\bar{q}^{(a, b)}(t)+\epsilon^{\prime} \sqrt{8 \pi G} Q(t),
$$

where $\bar{q}^{(a, b)}(t)$ is the solution to equation of motion given in eq. (3.22) and (3.23), $Q(t)$ is the fluctuation around the background $\bar{q}^{(a, b)}(t)$, and $\epsilon^{\prime}$ is the parameter to keep track of order of terms. This decomposition can be plugged back in total action given in (3.19) and (3.20) and expanded to second-order in $\epsilon^{\prime}$. $Q(t)$ obeys similar set of boundary conditions as the background $\bar{q}^{(a, b)}(t)$ :

$$
\begin{aligned}
& \text { Case (a): Specify } Q_{0} \text { and } \dot{Q}_{1} \Rightarrow Q_{0}=\dot{Q}_{1}=0, \\
& \text { Case (b) : Specify } \dot{Q}_{0} \text { and } Q_{1} \Rightarrow Q_{1}=\dot{Q}_{0}=0 .
\end{aligned}
$$

After imposing these boundary conditions on $Q$ and performing the expansion in powers of $\epsilon^{\prime}$ we notice that first order terms in $\epsilon^{\prime}$ vanish as $\bar{q}^{(a, b)}(t)$ satisfies equation of motion. The second order terms are non-vanishing. The series in $\epsilon^{\prime}$ stops at second order. The full expansion can be written as

$$
S^{(a, b)}=S_{\mathrm{tot}}^{(a, b)}-\frac{3 \epsilon^{\prime 2} V_{3}}{4 N_{c}} \int_{0}^{1} \mathrm{~d} t \dot{Q}^{2},
$$

where $S_{\text {tot }}^{(a, b)}$ is given in eq. (3.24) and (3.25). In the path-integral measure such a decomposition will imply

$$
\int \mathcal{D} q(t) \Rightarrow \int \mathcal{D} Q(t)
$$

As the action in eq. (4.4) separates into a part independent of $Q$ and part quadratic in $Q$, therefore the path-integral over $Q$ can be performed independently of the rest. This path-integral over $Q$ is

$$
F\left(N_{c}\right)=\frac{\int_{Q[0]=0}^{Q^{\prime}[1]=0} \mathcal{D} Q(t)}{\text { Case (a) }} \text { OR } \int_{Q^{\prime}[0]=0}^{Q[1]=0} \mathcal{D} Q(t) \exp \left(-\frac{3 i \epsilon^{\prime 2} V_{3}}{4 \hbar N_{c}} \int_{0}^{1} \mathrm{~d} t \dot{Q}^{2}\right) .
$$

This path-integral is very similar to the path-integral for a free where the trajectories at end points are kept fixed. However, this one is slightly different as at one of the boundary we are fixing $\dot{Q}$. A similar path-integral over mixed boundary conditions was encountered in [54] where the authors have computed it in appendix of the paper. Following the footsteps in [54] we note

$$
F\left(N_{c}\right)=\frac{1}{\sqrt{\pi i}}
$$


The important point to note is that in case of mixed boundary conditions the above pathintegrals leads a $N_{c}$-independent numerical factor, unlike in case of Dirichlet boundary conditions where the above path-integral is proportional to $N_{c}^{-1 / 2}$.

Then our transition amplitude $G\left[\mathrm{bd}_{0}, \mathrm{bd}_{1}\right]$ becomes

$$
G\left[\mathrm{bd}_{0}, \mathrm{bd}_{1}\right]=\frac{1}{\sqrt{\pi i}} \int_{0^{+}}^{\infty} \mathrm{d} N_{c} \exp \left(\frac{i}{\hbar} S_{\mathrm{tot}}^{(a, b)}\right),
$$

where $S_{\text {tot }}^{(a, b)}$ is given in eq. (3.24) and (3.25). Now the task is reduced to performing the contour integration over lapse $N_{c}$. Here we will make use of complex analysis and PicardLefschetz formalism to analyse this integral. We start by studying the various saddle points of the action $S_{\text {tot }}^{(a, b)}$ appearing in the exponent.

\subsection{Saddle points}

The saddle points of the action can be found using

$$
\frac{\partial S_{\mathrm{tot}}^{(a, b)}}{\partial N_{c}}=0
$$

The important thing to note here is about the structure of $S_{\text {tot }}^{(a, b)}$ in terms of $N_{c}$ which can be noticed from eq. (3.24) and (3.25). It has term proportional to $N_{c}^{3}, N_{c}, 1 / N_{c}$ and $1 / N_{c}^{3}$. Setting $(8 \pi G)=1$ the structural form for $S_{\text {tot }}^{(a, b)}$ can be written as

$$
S_{\mathrm{tot}}^{(a, b)}=\frac{V_{3}}{2}\left[A^{(a, b)} N_{c}+\frac{B^{(a, b)}}{N_{c}}+\frac{2 N_{c}^{3} \Lambda^{2}}{9}+\frac{\alpha C^{(a, b)}}{N_{c}^{3}}\right],
$$

where

$$
\begin{array}{ll}
A^{(a)}=6 k-\left(2 q_{0}+\dot{q}_{1}\right) \Lambda, & A^{(b)}=6 k+\left(\dot{q}_{0}-2 q_{1}\right) \Lambda, \\
B^{(a)}=\frac{3 \dot{q}_{1}\left(2 q_{0}+\dot{q}_{1}\right)}{2}+12 \alpha k \dot{q}_{1}, & B^{(b)}=\frac{3 \dot{q}_{1}\left(\dot{q}_{0}-2 q_{1}\right)}{2}-12 \alpha k \dot{q}_{0} \\
C^{(a)}=\dot{q}_{1}^{3}, & C^{(b)}=-\dot{q}_{0}^{3} .
\end{array}
$$

This structure is largely same as in the case of Einstein-Hilbert gravity, except the emergence of new additional term proportional to $1 / N_{c}^{3}$ which is coming from the Gauss-Bonnet sector. The presence of this new term give rise to additional saddle points which are absent in the case of Einstein-Hilbert gravity.

$$
\frac{\partial S_{\mathrm{tot}}^{(a, b)}}{\partial N_{c}}=0 \Rightarrow A^{(a, b)}-\frac{B^{(a, b)}}{N_{c}^{2}}+\frac{2 N_{c}^{2} \Lambda^{2}}{3}-\frac{3 \alpha C^{(a, b)}}{N_{c}^{4}}=0 .
$$

It can be seen from this that the saddle point equation is cubic in $N_{c}^{2}$, resulting in three pairs of roots. This cubic equation can be solved by the known methods of dealing with cubic polynomial equation. In particular if the cubic equation has real coefficients then the nature of roots can be determined by analysing the behaviour of the discriminant of 
cubic equation. Such a strategy is no longer valid if the coefficients are complex. The discriminant of cubic polynomial with real coefficients is given by

$$
\begin{aligned}
\Delta= & \left(A^{(a, b)}\right)^{2}\left(B^{(a, b)}\right)^{2}+\frac{8 \Lambda^{2}}{3}\left(B^{(a, b)}\right)^{2}+12 \alpha\left(A^{(a, b)}\right)^{3}\left(C^{(a, b)}\right) \\
& +36 \alpha \Lambda^{2}\left(A^{(a, b)}\right)\left(B^{(a, b)}\right)\left(C^{(a, b)}\right)-108 \alpha^{2} \Lambda^{4}\left(C^{(a, b)}\right)^{2} .
\end{aligned}
$$

If $\Delta>0$ the cubic equation has three distinct real roots for $N_{c}^{2}$. If $D<0$ the equation has two complex-conjugate roots, and one real root for $N_{c}^{2}$. By defining variables

$$
\begin{aligned}
& U=\frac{3}{4 \Lambda^{4}}\left(A^{(a, b)}\right)^{2}+\frac{3}{2 \Lambda^{2}} B^{(a, b)} \\
& V=\frac{3}{4 \Lambda^{4}} B^{(a, b)} A^{(a, b)}+\frac{1}{4 \Lambda^{6}}\left(A^{(a, b)}\right)^{3}-\frac{9 \alpha}{2 \Lambda^{2}} C^{(a, b)}
\end{aligned}
$$

one can write the roots as

$$
\begin{aligned}
& N_{0}^{ \pm}= \pm\left(y_{+}+y_{-}-\frac{1}{2 \lambda^{2}} A^{(a, b)}\right)^{1 / 2}, \\
& N_{1}^{ \pm}= \pm\left(y_{+} \omega+y_{-} \omega^{2}-\frac{1}{2 \lambda^{2}} A^{(a, b)}\right)^{1 / 2}, \\
& N_{2}^{ \pm}= \pm\left(y_{+} \omega^{2}+y_{-} \omega-\frac{1}{2 \lambda^{2}} A^{(a, b)}\right)^{1 / 2},
\end{aligned}
$$

where

$$
y_{ \pm}=\left(\frac{V}{2} \pm \sqrt{\frac{V^{2}}{4}-\frac{U^{3}}{27}}\right)^{1 / 3} .
$$

where $1, \omega$ and $\omega^{2}$ are the three roots of unity. These are the six saddle points that arise in this system.

The boundary conditions decide the nature of $A^{(a, b)}, B^{(a, b)}$ and $C^{(a, b)}$. If they are real then one can compute the discriminant of the cubic equation whose behaviour dictates the kind of roots for $N_{c}^{2}$. We can collectively write the saddle point as $N_{\sigma}^{ \pm}$, where $\sigma=0,1$, and 2. Corresponding to each of these saddle points we have a metric

$$
\left(\mathrm{d} s_{\sigma}^{(a, b)}\right)^{2}=-\frac{N_{\sigma}^{2}}{q^{(a, b)}(t)} \mathrm{d} t^{2}+q^{(a, b)}(t)\left[\frac{\mathrm{d} r^{2}}{1-k r^{2}}+r^{2} \mathrm{~d} \Omega_{2}^{2}\right],
$$

where $q^{(a, b)}(t)$ is given by eq. (3.22) and (3.23). Note that it is $N_{\sigma}^{2}$ that enters the metric, which implies that the metric is same for each pair $N_{\sigma}^{ \pm}$of saddle points. As long as $N_{\sigma}^{2}$ is real and positive, we are in Lorentzian signature. When it is real and negative then it is Euclidean signature, as in those cases $N_{\sigma}$ is imaginary. In cases when $N_{\sigma}^{2}$ is complex, the spacetime has a mixed signature. Geometries become singular when $q^{(a, b)}(t) \rightarrow 0$. In this case the spacetime volume goes to zero.

For each of these saddle points one has a corresponding on-shell action. As the saddle points will generically be complex in nature therefore their corresponding on-shell action 
will have a real and an imaginary part. The momentum at the saddles can be computed using eq. (3.21).

$$
\pi^{(a, b)}=\frac{3 \dot{q}^{(a, b)}}{N_{\sigma}^{ \pm}} .
$$

By making use of solution to equation of motion given in (3.22) and (3.23) one can compute the momentum at the end points.

$$
\begin{array}{rlrl}
\pi_{0}^{(a)} & =\frac{3}{N_{\sigma}^{(a) \pm}}\left[\dot{q}_{1}-\frac{2 \Lambda N_{\sigma}^{(a) 2}}{3}\right], & \pi_{1}^{(a)}=\frac{3 \dot{q}_{1}}{N_{\sigma}^{(a) \pm}} \\
\pi_{0}^{(b)}=\frac{3 \dot{q}_{0}}{N_{\sigma}^{(b) \pm}}, & \pi_{1}^{(b)}=\frac{3}{N_{\sigma}^{(b) \pm}}\left(\frac{2 \Lambda N_{\sigma}^{(b) 2}}{3}+\dot{q}_{0}\right) .
\end{array}
$$

The crucial point to note here is that momentum at the boundaries can be complex if the saddle point $N_{\sigma}$ is complex. This is interesting as it carries characteristics of tunneling phenomena.

\section{$5 \quad N_{c}$-integration via Picard-Lefschetz}

We then go forth to compute the $N_{c}$-integration. We will make use of Picard-Lefschetz (PL) theory to analyse the behavior of the integrand in the complex plane [55-58]. Along with PL theory we make use of WKB methods to compute the integral. For this we need the set of saddle points and collection of steepest descent/ascent paths associated with each saddle point. A saddle point is termed 'relevant' if the steepest ascent path emanating from it intersects the original contour integration. The original integration contour can then be distorted to lie along the steepest descent paths passing through relevant saddle points. Instead of using the prescription of Wick-rotation to deform the contour, we follow the methods of PL-theory to choose a contour of integration uniquely, along which the integrand is absolutely convergent.

The problem of performing path-integration is reduced to a task of computing thimbles (steepest descent paths) on a complex plane. In the following we will give a review of Picard-Lefschetz formalism. We start by considering the path-integral in the following manner

$$
I=\int \mathcal{D} z(t) e^{i \mathcal{S}(z) / \hbar}
$$

where the exponent is functional of $z(t)$. In general the integrand can be quite oscillatory and hence not an easy task to compute the integral. In flat spacetime the global symmetries of spacetime allow one to cast Lorentz group in to a compact rotation group under a transformation of time co-ordinate. This privilege doesn't exist in non-flat spacetimes. Such a transformation of time co-ordinate in flat spacetime leads to exponential damping of above integrand. In PL theory one analytically continues both $z(t)$ and $\mathcal{S}(z)$ in to complex plane, and interprets $\mathcal{S}$ as an holomorphic functional of $z(t)$. This implies that $\mathcal{S}$ satisfies a functional form of Cauchy-Riemann conditions

$$
\frac{\delta \mathcal{S}}{\delta \bar{z}}=0 \Rightarrow \begin{cases}\frac{\delta \operatorname{Re} \mathcal{S}}{\delta x} & =\frac{\delta \operatorname{Im} \mathcal{S}}{\delta y} \\ \frac{\delta \operatorname{Re} \mathcal{S}}{\delta y} & =-\frac{\delta \operatorname{Im} \mathcal{S}}{\delta x}\end{cases}
$$




\subsection{Flow equations}

On writing the complex exponential as $\mathcal{I}=i \mathcal{S} / \hbar=h+i H$ and writing $z(t)=x_{1}(t)+i x_{2}(t)$, the downward flow is defined as

$$
\frac{\mathrm{d} x_{i}}{\mathrm{~d} \lambda}=-g_{i j} \frac{\partial h}{\partial x_{j}},
$$

where $g_{i j}$ is a metric defined on the complex manifold, $\lambda$ is flow parameter and $(-)$ sign refers to downward flow. The steepest descent flow lines follow a trajectory dictated by above equation. They are also knowns as thimbles (can be denoted by $\mathcal{J}_{\sigma}$ ). Steepest ascent flow lines are defined with a plus sign in front of $g_{i j}$ in the eq. (5.3), and are denoted as $\mathcal{K}_{\sigma}$. Here $\sigma$ refers to the saddle point to which these flow-lines are attached. The definition of flow lines immediately implies that the real part $h$ (also called Morse function) decreases monotonically as one moves away from the critical point along the steepest descent curves. This can be seen by computing

$$
\frac{\mathrm{d} h}{\mathrm{~d} \lambda}=g_{i j} \frac{\mathrm{d} x^{i}}{\mathrm{~d} \lambda} \frac{\partial h}{\partial x_{j}}=-\left(\frac{\mathrm{d} x_{i}}{\mathrm{~d} \lambda} \frac{\mathrm{d} x^{i}}{\mathrm{~d} \lambda}\right) \leq 0 .
$$

This generically holds for any Riemannian metric. However, in this paper for simplicity we assume $g_{z, z}=g_{\bar{z}, \bar{z}}=0$ and $g_{z, \bar{z}}=g_{\bar{z}, z}=1 / 2$. This leads to a simplified version of flow equations

$$
\frac{\mathrm{d} z}{\mathrm{~d} \lambda}= \pm \frac{\partial \overline{\mathcal{I}}}{\partial \bar{z}}, \quad \frac{\mathrm{d} \bar{z}}{\mathrm{~d} \lambda}= \pm \frac{\partial \mathcal{I}}{\partial z}
$$

Using them it is easy to notice that the imaginary part of $\operatorname{Im} \mathcal{I}=H$ is constant along the flow lines.

$$
\frac{\mathrm{d} H}{\mathrm{~d} \lambda}=\frac{1}{2 i} \frac{\mathrm{d}(\mathcal{I}-\overline{\mathcal{I}})}{\mathrm{d} \lambda}=\frac{1}{2 i}\left(\frac{\partial \mathcal{I}}{\partial z} \frac{\mathrm{d} z}{\mathrm{~d} \lambda}-\frac{\partial \overline{\mathcal{I}}}{\partial \bar{z}} \frac{\mathrm{d} \bar{z}}{\mathrm{~d} \lambda}\right)=0 .
$$

This is a wonderful feature of flow-lines and can be used to determine the structure of flowlines in the complex $N_{c}$-plane. It is seen that the oscillatory integral becomes convergent and well-behaved along any of the steepest descent lines (thimbles). This motivates one check if it is possible to analytically deform the original integration contour to integration along either one thimble or a sum of thimbles. This is a true generalization of Wick-rotation.

In the complex $N_{c}$-plane the flow equations corresponding to steepest descent (ascent) becomes the following in cartesian co-ordinates

$$
\begin{aligned}
\text { Descent } \Rightarrow & \frac{\mathrm{d} x_{1}}{\mathrm{~d} \lambda}=-\frac{\partial \operatorname{Re} \mathcal{I}}{\partial x_{1}}, & \frac{\mathrm{d} x_{2}}{\mathrm{~d} \lambda}=-\frac{\partial \operatorname{Re} \mathcal{I}}{\partial x_{2}}, \\
\text { Ascent } \Rightarrow & \frac{\mathrm{d} x_{1}}{\mathrm{~d} \lambda}=\frac{\partial \operatorname{Re} \mathcal{I}}{\partial x_{1}}, & \frac{\mathrm{d} x_{2}}{\mathrm{~d} \lambda}=\frac{\partial \operatorname{Re} \mathcal{I}}{\partial x_{2}} .
\end{aligned}
$$

It is noticed that the $\operatorname{Im} \mathcal{I}$ doesn't enter the flow equations as $\operatorname{Im} \mathcal{I}=$ const. along the flow lines. Each saddle point has two steepest descent lines and two steepest ascent lines attached to it. The boundary conditions and the parameter values dictate the location of the saddles on the complex $N_{c}$-plane. Solving these flow equations can be sometimes hard as $\mathcal{I}$ can be complicated. However, it is possible to deal with them numerically. One can bypass solving them entirely by making use of knowledge that $H$ is constant along 
them. This determines all the flow-lines. But to find out about the nature of flow lines one has to compute the gradient of first derivative (second order derivative of action at the saddle points).

\subsection{Choice of contour}

Once the set of saddle points along with the set of steepest ascent/descent flow-lines associated with each saddle point are known, one can begin to find the new contour of integration to which the original integration contour will be deformed. The integral in complex $N_{c^{-}}$ plane is absolutely convergent along this new contour (for more detail see $[38,56,57]$ ).

In the complex $N_{c}$ plane the behavior of $h$ and $H$ determines the 'allowed' regions (region where integral is well-behaved) and 'forbidden' region (region where integral diverges). We label the former by $J_{\sigma}$ while later is denoted by $K_{\sigma}$, and as mentioned previously $\sigma$ refers the saddle point. These regions have $h\left(J_{\sigma}\right)<h\left(N_{\sigma}\right)$, while $h\left(K_{\sigma}\right)>h\left(N_{\sigma}\right) . h$ goes to $-\infty$ along the steepest descent lines and ends in a singularity, while along the steepest ascent contours $h \rightarrow+\infty$. These lines usually intersect at only one point where they are both well-defined. With a suitable choice of orientation one can write

$$
\operatorname{Int}\left(\mathcal{J}_{\sigma}, \mathcal{K}_{\sigma^{\prime}}\right)=\delta_{\sigma \sigma^{\prime}} .
$$

The purpose is to write the integral over the original contour as an integral along the new contour which is sum of integrations done along Lefschetz thimbles. Schematically this can be expressed as

$$
\mathbb{D}=\left(0^{+}, \infty\right) \Rightarrow \mathcal{C}=\sum_{\sigma} n_{\sigma} \mathcal{J}_{\sigma},
$$

in a homological sense for some integers $n_{\sigma}$ which will take value 0 or \pm 1 when accounting for orientation of contour over each thimble. This will also imply that $n_{\sigma}=\operatorname{Int}\left(\mathcal{C}, \mathcal{K}_{\sigma}\right)=$ $\operatorname{Int}\left(\mathbb{D}, \mathcal{K}_{\sigma}\right)$. As the intersection number is topological and doesn't change if we deform the contour, therefore the necessary and sufficient condition for a thimble $\mathcal{J}_{\sigma}$ to be relevant is that the steepest ascent curve from the corresponding saddle point intersects the original integration domain $\mathbb{D}$. The integration contour is chosen to lie in the region $J_{\sigma}$ (which is the 'allowed' region) and follow the contour trajectory dictated by the steepest descent paths [38]. In this circumstance there is no hindrance in smoothly sliding the intersection point along the $\mathcal{K}_{\sigma}$ to the relevant saddle point.

Once the original integration contour is deformed to a sum over integration done along various relevant thimbles then we have

$$
I=\int_{\mathcal{C}} \mathrm{d} z(t) e^{i S[z] / \hbar}=\sum_{\sigma} n_{\sigma} \int_{\mathcal{J}_{\sigma}} \mathrm{d} z(t) e^{i S[z] / \hbar} .
$$

It is common that in such process more than one thimble contributes to integration, resulting in interference of contributions coming from various thimbles. This is feature of performing complex integration via Picard-Lefschetz methodology. The integration along each of the thimbles is absolutely convergent if

$$
\left|\int_{\mathcal{J}_{\sigma}} \mathrm{d} z(t) e^{i S[z] / \hbar}\right| \leq \int_{\mathcal{J}_{\sigma}}|\mathrm{d} z(t)|\left|e^{i S[z] / \hbar}\right|=\int_{\mathcal{J}_{\sigma}}|\mathrm{d} z(t)| e^{h}(z)<\infty .
$$


If we denote the length along the contour path as $l=\int|\mathrm{d} z(t)|$, then convergence of above integral require that $e^{h} \sim 1 / l$ as $l \rightarrow \infty$. The original integration hence can be analytically deformed into a sum of absolutely convergent integrals along various Lefschtez thimbles passing through relevant saddle points. If one does an expansion in $\hbar$ then to leading order we get the following

$$
I=\int_{\mathcal{C}} \mathrm{d} z(t) e^{i S[z] / \hbar}=\sum_{\sigma} n_{\sigma} e^{i H\left(N_{\sigma}\right)} \int_{\mathcal{J}_{\sigma}} \mathrm{d} z(t) e^{h} \approx \sum_{\sigma} n_{\sigma} e^{i S\left[N_{\sigma}\right] / \hbar}\left[\mathcal{A}_{\sigma}+\mathcal{O}(\hbar)\right],
$$

where $\mathcal{A}_{\sigma}$ is the contribution coming after performing a gaussian integration around the saddle point $N_{\sigma}$.

\subsection{Flow directions}

The direction of flow lines either emanating from the saddles or going into it can be determined analytically (to some extent) by expanding the $N_{c}$-action of theory given in eq. (3.24) and (3.25) around the saddle points given in eq. (4.17). If we write $N_{c}=N_{\sigma}+\delta N$ (where $N_{\sigma}$ is any saddle point of action), then the action has a power series expansion in $\delta N$.

$$
S^{(0)}=S_{\sigma}^{(0)}+\left.\frac{\mathrm{d} S^{(0)}}{\mathrm{d} N_{c}}\right|_{N=N_{\sigma}} \delta N+\left.\frac{1}{2} \frac{\mathrm{d}^{2} S^{(0)}}{\mathrm{d} N_{c}^{2}}\right|_{N=N_{\sigma}}(\delta N)^{2}+\cdots .
$$

The first order terms will vanish identically by definition.

The second order terms can be computed directly from the action in eq. (3.24) and (3.25), by just taking double-derivative with respect to $N_{c}$. From this the direction of flow-lines can be determined. One should recall that the imaginary part of exponential $i S$ (or $H$ ) is constant along the flow lines. This implies that $\operatorname{Im}\left[i S-i S\left(N_{s}\right)\right]=0$. The second variation at the saddle point can be written as $\mathrm{d}^{2} S^{(0)} / \mathrm{d} N_{c}^{2}=r e^{i \rho}$, where $r$ and $\rho$ depends on boundary conditions. Near the saddle point the change in $H$ will go like

$$
\Delta(H) \propto i\left(\left.\frac{\mathrm{d}^{2} S^{(0)}}{\mathrm{d} N^{2}}\right|_{N_{\sigma}}\right)\left(\delta N_{c}\right)^{2} \sim n_{\sigma}^{2} e^{i\left(\pi / 2+2 \theta_{\sigma}+\rho_{\sigma}\right)},
$$

where we write $\delta N=n_{\sigma} e^{i \theta_{\sigma}}$ and $\theta_{\sigma}$ is the direction of flow lines at the corresponding saddle point. Given that the imaginary part $H$ remains constant along the flow lines, so this means

$$
\theta_{\sigma}=\frac{(2 k-1) \pi}{4}-\frac{\rho_{\sigma}}{2}
$$

where $k \in \mathbb{Z}$.

For the steepest descent and ascent flow lines, their corresponding $\theta_{\sigma}^{\text {des/aes }}$ is such that the phase for $\Delta H$ correspond to $e^{i\left(\pi / 2+2 \theta_{\sigma}+\rho_{\sigma}\right)}=\mp 1$. This implies

$$
\theta_{\sigma}^{\text {des }}=k \pi+\frac{\pi}{4}-\frac{\rho_{\sigma}}{2}, \quad \theta_{\sigma}^{\text {aes }}=k \pi-\frac{\pi}{4}-\frac{\rho_{\sigma}}{2} .
$$

These angles can be computed numerically for the given boundary conditions and for gravitational actions. 


\subsection{Saddle-point approximation}

Once the set of saddle points, flow directions and steepest descent/ascent paths associated with them (denoted by $\mathcal{J}_{\sigma} / \mathcal{K}_{\sigma}$ respectively) are known, it is then easy to find the relevant saddle points. A saddle point is termed relevant if the steepest ascent path emanating from it intersect with the original contour of integration. In the current case the original integration contour is $\left(0^{+}, \infty\right)$. The original integration contour then becomes sum over the contribution coming from all the Lefschetz thimbles passing through relevant saddle points. We can then do saddle-point-approximation to compute the transition amplitude in eq. (4.8). In the $\hbar \rightarrow 0$ limit we have

$$
G\left[\mathrm{bd}_{0}, \mathrm{bd}_{1}\right] \approx \frac{1}{\sqrt{\pi i}} \sum_{\sigma} n_{\sigma} \exp \left[\frac{i}{\hbar} S_{\text {tot }}^{(a, b)}\left(N_{\sigma}\right)\right] \int_{\mathcal{J}_{\sigma}} \mathrm{d} N_{c} \exp \left[\frac{i}{\hbar}\left(S_{\text {tot }}^{(a, b)}\right)_{N_{c} N_{c}}\left(N_{c}-N_{\sigma}\right)^{2}\right],
$$

where we consider only the leading order term in $\hbar$. Here $N_{\sigma}$ are the relevant saddle points for the various boundary conditions given in eq. (4.17), $S_{\text {tot }}^{(a, b)}\left(N_{\sigma}\right)$ is the on-shell action which can be computed from eq. (3.24) and (3.25) for the relevant saddles. $\left(S_{\text {tot }}^{(a, b)}\right)_{N_{c} N_{c}}$ is the second variation of the action with respect to $N_{c}$ computed at the relevant saddle points.

On writing $N-N_{\sigma}=n e^{i \theta_{\sigma}}$, where $\theta_{\sigma}$ is the angle the Lefschetz thimble make with the real $N$-axis while $\sigma$ corresponds to relevant saddle point. Then the above integration can be performed easily. It gives the following

$$
G\left[q_{0}, q_{1}\right]=\frac{1}{\sqrt{\pi i}} \sum_{\sigma} n_{\sigma}\left|\left(S_{\text {tot }}^{(a, b)}\right)_{N_{c} N_{c}}\right|^{-1 / 2} \exp \left[i \theta_{\sigma}+\frac{i}{\hbar} S_{\text {tot }}^{(a, b)}\left(N_{\sigma}\right)\right] .
$$

In the next section we will make use of it and apply it to the case of no-boundary proposal of Universe to compute the transition amplitude.

\section{$6 \quad$ No-boundary Universe}

This is special boundary condition where the Universe start from nothing. In the current situation this implies $q_{0}=0$, implying that Universe started with a zero scale factor $a[36,38-40,49,50]$. In case $(a)$ where we specify field $q_{0}$ at one end point while its first derivative $\dot{q}_{1}$ at another end point this immediately leads to a simplified $N_{c}$ action. However, at the final boundary, following the solution to equation of motion from eq. (3.22) we also have a relation

$$
\dot{q}_{1}^{(a)}=q_{1}^{(a)}+\frac{\Lambda N_{c}^{2}}{3} .
$$

This allow us to express the derivative of field in terms of field value at the final boundary. This is useful as one can study the problem by doing the analysis in terms of $q_{1}$ where we require that for physical reasons $q_{1}>0$. A real and positive $q_{1}$ immediately implies a possibly complex $\dot{q}_{1}^{(a)}$ if $N_{c}^{2}$ is complex. On the other hand this also means that if $q_{1}$ has a fixed real positive value and the number of relevant saddle points are more that one, then it will imply that at the final boundary $\dot{q}_{1}^{(a)}$ will have multiple values. This is contradictory to our initial requirement that in case $(a) \dot{q}_{1}^{(a)}$ is fixed at final boundary, and implies that the Universe at final time has multiple values of $\dot{q}_{1}^{(a)}$. 
In the case $(b)$ on other hand the boundary conditions require fixing $\dot{q}_{0}^{(b)}$ and $q_{1}^{(b)}$. This implies that at $t=0$ by following the solution 3.23 , if the Universe started from nothing $\left(q^{(b)}(t=0)=0\right)$, then it leads to a relationship between $\dot{q}_{0}^{(b)}$ and $q_{1}^{(b)}$

$$
\dot{q}_{0}^{(b)}=q_{1}^{(b)}-\frac{\Lambda N_{c}^{2}}{3} .
$$

Here if at final boundary $q_{1}^{(b)}>0$ (real and positive) then the initial $\dot{q}_{0}^{(b)}$ could be complex when $N_{c}^{2}$ is complex. Moreover, when the number of relevant saddle points are more than one, then the final geometry is seen to arising from superposition multiple initial geometries. This is quite possible and doesn't lead to a contradiction unlike in case $(a)$. In the following we will study this particular scenario in more detail. We can plug the relation in eq. (6.2) in the action for $N_{c}$ for case (b) in eq. (3.25) to obtain the $N_{c}$-action for the no-boundary proposal.

$$
\begin{aligned}
S_{\mathrm{tot}}^{(b)}= & \frac{V_{3}}{16 \pi G}\left[6 k N_{c}+\frac{\left\{(\alpha \Lambda-3) q_{1}+12 k \alpha\right\} N_{c} \Lambda}{3}+\frac{(9+2 \alpha \Lambda) q_{1}^{2}+24 k \alpha q_{1}}{2 N_{c}}\right. \\
& \left.+\frac{(3+2 \alpha \Lambda) \Lambda^{2} N_{c}^{3}}{54}+\frac{\alpha q_{1}^{3}}{N_{c}^{3}}\right] .
\end{aligned}
$$

It should be noted that if we set $\alpha=0$ then we get the action for the no-boundary Universe in case of pure Einstein-Hilbert gravity. We note that this residual action is bit different from the action that one obtains in the case of dirichlet boundary conditions [38-40]. This is because we used mixed boundary conditions to arrive at the action in eq. (6.3). The saddle point equation correspondingly is

$$
\begin{aligned}
\text { Case (b) : } \quad & \frac{\Lambda^{2}(3+2 \alpha \Lambda)}{18} N_{c}^{6}+\left\{6 k-q_{1} \Lambda+\frac{\alpha \Lambda\left(12 k+q_{1} \Lambda\right)}{3}\right\} N_{c}^{4} \\
& -\left\{12 k \alpha q_{1}+\left(\frac{9}{2}+\alpha \Lambda\right) q_{1}^{2}\right\} N_{c}^{2}-3 \alpha q_{1}^{3}=0 .
\end{aligned}
$$

The interesting thing to note here is that in case $(b)$ there exist a $q_{1}$ for which the coefficient of $N_{c}^{4}$ in eq. (6.4) can vanishe. This will offer some simplification in the expressions for saddle points.

The saddle-point equation is cubic in $N_{c}^{2}$ with real coefficients. Its nature of roots can be decided based by analysing the behaviour of its discriminant in the parameter space of couplings and boundary value $q_{1}$. It is seen that for positive $k, \Lambda$, and $\alpha$ the discriminant is always positive for $q_{1} \geq 0$. This is interesting as it quickly implies that the saddle point equation has three distinct real roots for $N_{c}^{2}$. Also as $\alpha$ and $q_{1}$ are positive, the zeroth-order term in $N_{c}^{2}$ in the saddle-point equation is positive. This means that the product of three roots has to be positive. It leads to two possibilities: either all roots for $N_{c}^{2}$ are positive or one is positive and other two are negative. However, as the coefficient of $N_{c}^{2}$ is negative so this immediately implies the later case with one positive root and two negative roots for $N_{c}^{2}$. This means that we have two saddle points lying on real-axis in complex $N_{c}$-plane (one positive and one negative); while four saddle points lie on imaginary axis in complex 
$N_{c}$-plane (two of them in positive imaginary axis, while other two in negative imaginary axis). It is worth stating here that saddle point where $N_{c}^{2}>0$ correspond to the usual Lorentzian geometry with a Lorentzian evolution, as can be seen from eq. (4.19). On the other hand the saddle point where $N_{c}^{2}<0$ correspond to Euclidean geometries.

To workout the transition amplitude in eq. (5.18) one also needs to know the second variation of the above action. These will subsequently be needed to determine the direction of Lefschetz thimbles at the various relevant saddle points. The second variation is given by

$$
\left(S^{(b)}\right)_{, N_{c} N_{c}}=\frac{V_{3}}{16 \pi G}\left[\frac{(3+2 \alpha \Lambda) \Lambda^{2} N_{c}}{9}+\frac{(9+2 \alpha \Lambda) q_{1}^{2}+24 k \alpha q_{1}}{N_{c}^{3}}+\frac{12 \alpha q_{1}^{3}}{N_{c}^{5}}\right] .
$$

At real saddle points the second variation is also real, while when $N_{c}$ is complex then the second variation will also be complex.

The nature of relevance of each of these saddle-points depends on the parameter values and whether the steepest ascent path emanating from them intersects the original integration contour. In principle this seems like a well-defined way of finding out the relevance of saddle points. However, in practice often the action has large amount of symmetry. Due to this there is degeneracy between steepest ascent and steepest descent curves. It means that the steepest ascent curve from one saddle point overlaps with the steepest descent curve from another saddle point. To lift these degeneracy one can add a small perturbation in the $N_{c}$ action which helps in breaking symmetry. Lifting this degeneracy also aid us to correctly locate the relevant saddle points.

We will consider a numerical example to investigate the state of art once parameters are fixed to some value. For numerical analysis and to lift degeneracy of the system we consider adding a small perturbation to the $N_{c}$ action

$$
S_{\text {pert }}=\frac{i V_{3} \epsilon^{\prime \prime} N_{c}}{16 \pi G}
$$

where $\epsilon^{\prime \prime}$ is a small parameter. Notice that the perturbation is imaginary in nature and it is somewhat reminiscent to $+i \epsilon$-prescription in standard flat spacetime field theory. In a sense we are inspired by the Feynman's $+i \epsilon$-prescription to choose this perturbation.

For purpose of better understanding the system, we pick up an example. We consider the value of parameters: $k=1, \Lambda=3, \alpha=2$ and $\epsilon^{\prime \prime}=10^{-2}$ (we have set $8 \pi G=1$ ). For $k=1$ the volume $V_{3}=\pi^{2}$ which can be computed using eq. (2.8). We compute the saddle points following the eq. (6.4). As discussed previously it is seen that the cubic equation in $N_{c}^{2}$ has three distinct roots: $N_{0}^{2}, N_{1}^{2}$ and $N_{2}^{2}$. As expected the $\operatorname{Re}\left(N_{0}^{2}\right)>0, \operatorname{Re}\left(N_{1}^{2}\right)<0$ and $\operatorname{Re}\left(N_{2}^{2}\right)<0$ for all $q_{1}$. Each of these roots for $N_{c}^{2}$ gets a small imaginary part due to the perturbation, which thereby give rise to a small deviation in the saddle points value

$$
\begin{array}{ll}
\operatorname{Im}\left(\delta N_{0}^{ \pm}\right)=\mp \nu_{0}, & \nu_{0}>0, \\
\operatorname{Re}\left(\delta N_{1}^{ \pm}\right)= \pm \nu_{1}, & \nu_{1}>0, \\
\operatorname{Re}\left(\delta N_{2}^{ \pm}\right)= \pm \nu_{2}, & \nu_{2}>0 .
\end{array}
$$

In figure 1 we plot the real and imaginary part of these roots as a function of $q_{1}$ (the value of $q$ at $t=1$ ). To determine the nature of saddle points (relevant or irrelevant) one has 

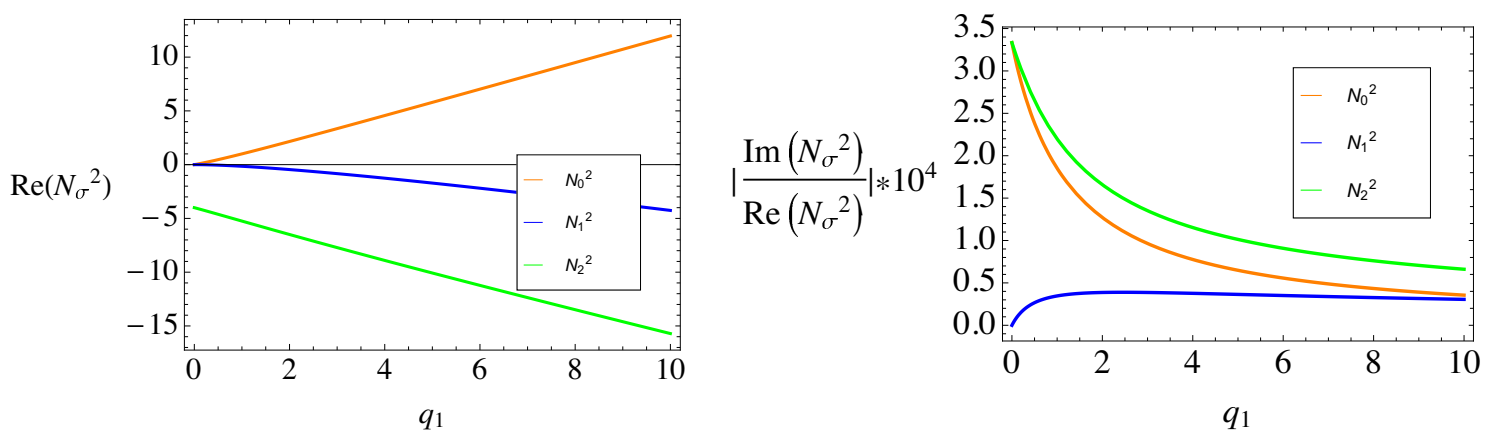

Figure 1. Here we analyse the real and imaginary part of the saddle points $\left(N_{0}^{2}, N_{1}^{2}\right.$ and $\left.N_{2}^{2}\right)$ for various values of $q_{1}$. In this numerical example we consider $k=1, \Lambda=3, \alpha=2$ and $\epsilon^{\prime \prime}=10^{-2}$. There is a small imaginary part which comes due to the perturbation added to the total $N_{c}$-action. As discussed the real part of $N_{0}^{2}$ remains positive, while real part of $N_{1}^{2}$ and $N_{2}^{2}$ remains always negative. In the plot on left we show the real part of various $N_{\sigma}^{2}$, while on the right plot we see the behavior of $\left|\operatorname{Im}\left(N_{\sigma}^{2}\right) / \operatorname{Re}\left(N_{\sigma}^{2}\right)\right|$ as a function of $q_{1}$. For the plot on right we have scaled the value by $10^{4}$ to plotting purpose.
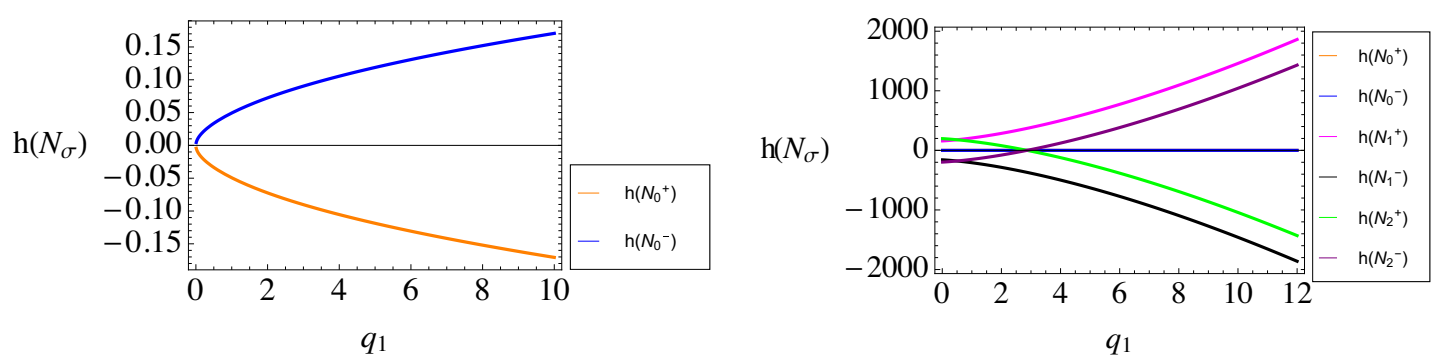

Figure 2. Plotting Morse-function $h$ for various saddle point against $q_{1}$. For this we consider parameter values $k=1, \Lambda=3, \alpha=2$ and $\epsilon^{\prime \prime}=10^{-2}$. From the plots we notice that only $h\left(N_{0}^{-}\right)$ and $h\left(N_{1}^{-}\right)$remains always negative. $h\left(N_{2}^{ \pm}\right)$change sign after a certain threshold $q_{1}^{\text {th }}$.

to study the steepest descent/ascent flow lines corresponding to each saddle point. These flow lines can be drawn by exploiting the knowledge that along these lines $H\left(N_{c}\right)=H\left(N_{\sigma}\right)$. In the absence of perturbation in (6.6) there will be some degeneracy in the sense that steepest descent line of one saddle will overlap with the steepest ascent line from another saddle. The addition of perturbation helps in lifting this degeneracy. In order to find the relevance of saddle points it is also crucial to analyse the nature of Morse-function $h$ at each of these saddle points. Picard-Lefschetz theory dictates that relevant saddle points must be reached by flowing down from the original integration contour via steepest ascent paths. This will immediately imply that $h<0$ at the relevant saddle points. A complex action bypasses this rigid constraint though. However, in our present case this is not possible.

If we plot $h\left(N_{\sigma}\right)$ against $q_{1}$ we notice that for some of saddle points $h$ changes sign as $q_{1}$ is varied. This is shown in figure 2. For the present situation there are six saddle points. Only those which can be reached by flowing downward along the steepest ascent lines from the original integration contour are relevant. The saddle points $N_{0}^{+}$and $N_{0}^{-}$lie in lower-right and upper-left quadrant respectively. Only the former can be reached via 


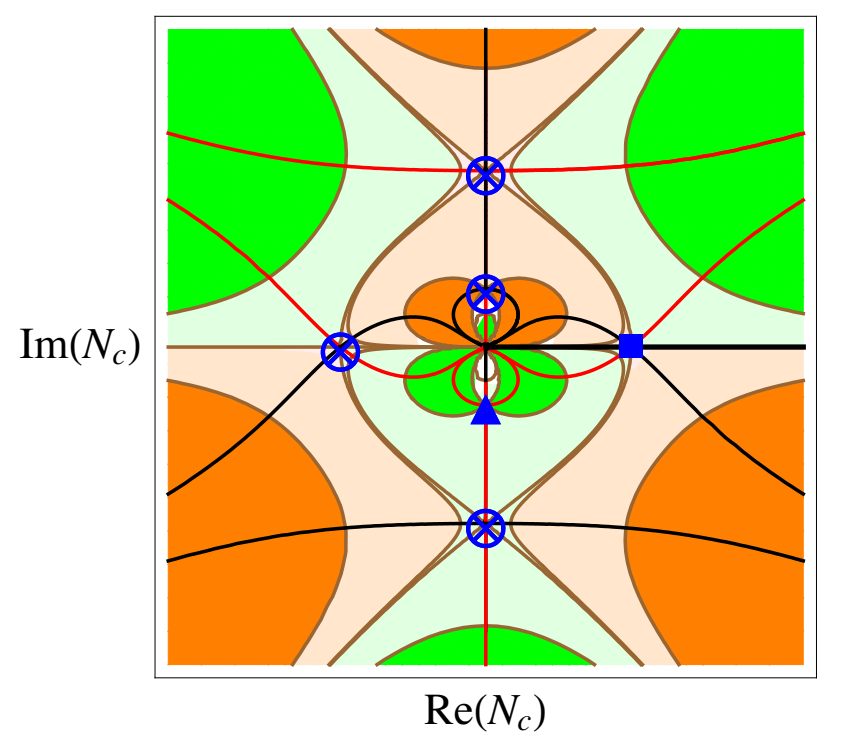

Figure 3. We consider the case of no-boundary Universe where we choose parameter values: $k=1$, $\Lambda=3, \epsilon^{\prime \prime}=10^{-2}$ and $\alpha=2$. We take $q_{1}=3$. We plot on $x$-axis real-part of $N_{c}$ while the $y$-axis is imaginary part of $N_{c}$. The red lines correspond to steepest descent lines (thimbles $\mathcal{J}_{\sigma}$ ), while the thin black lines are steepest ascent lines and denoted by $\mathcal{K}_{\sigma}$. The various saddle points $N_{\sigma}$ are shown in blue. The blue cross-circle are irrelevant saddle points. The saddle point blue-square and blue-triangle are relevant. The steepest ascent line emanating from it can be connected to original integration contour. $H$ remains constant along the red and black lines, and is equal to the value of $H\left(N_{\sigma}\right)$. The green region is allowed region with $h<h\left(N_{\sigma}\right)$ for all values of $\sigma$. The orange region (forbidden region) has $h>h\left(N_{\sigma}\right)$ for all $\sigma$. The light-green, light-orange and un-colored region has intermediary values. The boundary of these region is depicted in brown lines. Along these line we have $h=h\left(N_{\sigma}\right)$. The original contour of integration $\left(0, \infty^{+}\right)$is shown by thick black line.

steepest ascent lines from original integration contour and hence is relevant. The saddles $N_{1}^{+}$and $N_{1}^{-}$lie in upper-right and lower-left quadrant respectively. Both these saddlepoint can be reached from original integration contour by flowing downward along the steepest ascent flow lines. However only the later lie in allowed region with corresponding $h<0$ and is favourable. $N_{1}^{-}$is therefore relevant. The saddle points $N_{2}^{+}$and $N_{2}^{-}$lie in lower-right and upper-left quadrant respectively. Both are irrelevant: the former can't be reached via a steepest ascent path from original integration contour while later has $h>0$. So out of six saddle-points only two of them are relevant: $N_{0}^{+}$and $N_{1}^{-}$. In figure 3 we consider an example of the above scenario. We plot the set of saddle points along with the collection of flow lines associated with each saddle. The coloring of graph is done obeying the relation between the values of Morse-function at various saddle points. The region where $h\left(N_{c}\right)<h\left(N_{1}^{-}\right)$is colored green. The region where $h\left(N_{c}\right)>h\left(N_{1}^{+}\right)$ is colored orange. The light-green and light-orange region has intermediary values. The boundary of these regions is depicted in brown lines. The steepest descent lines are shown in red while the steepest ascent lines are shown in black. The thick black-line depicts the original integration contour. The upward flow through original integration contour only hits the saddle points $N_{0}^{+}, N_{1}^{-}$and $N_{2}^{-}$. However, only the former two are relevant with 


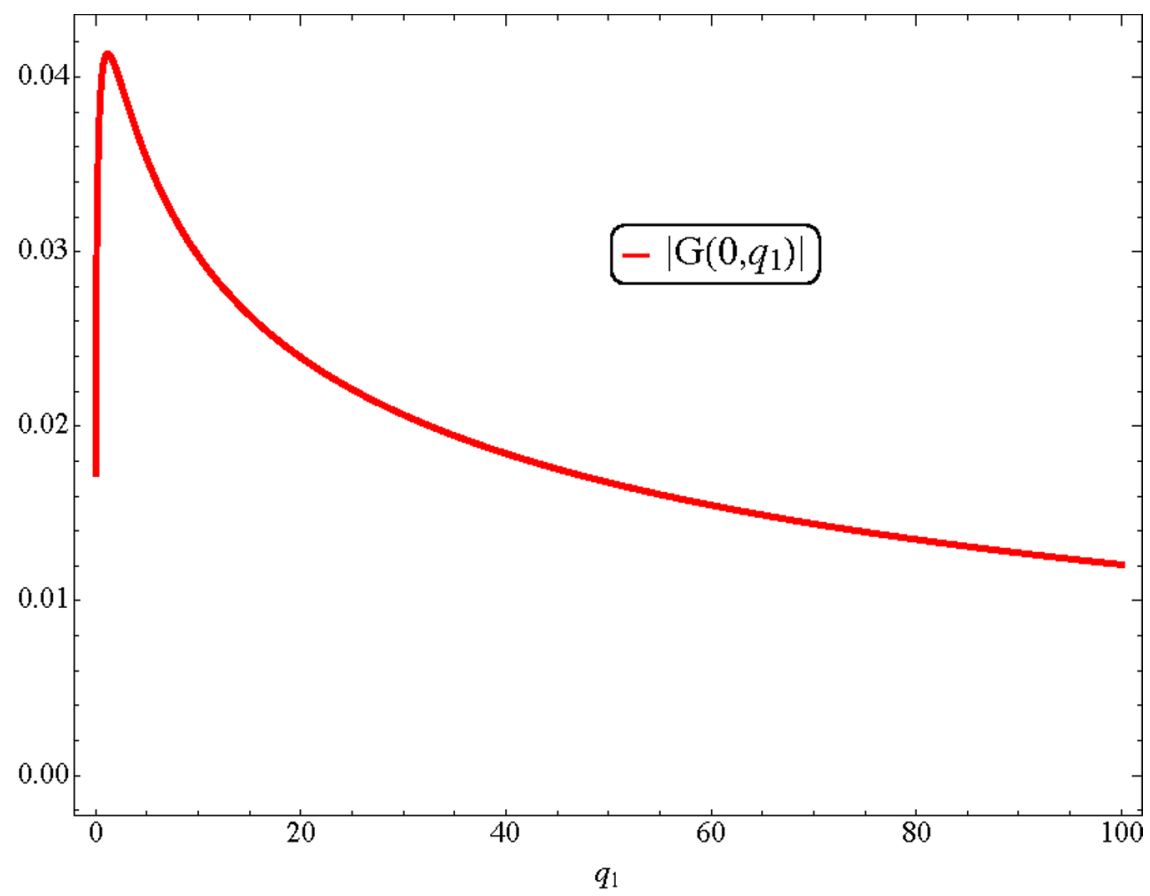

Figure 4. We consider the case of no-boundary Universe where we choose parameter values: $k=1$, $\Lambda=3, \epsilon^{\prime \prime}=10^{-2}$ and $\alpha=2$. Here we plot the transition amplitude $G\left(0, q_{1}\right)$ as $q_{1}$ is varied.

corresponding $h<0$, while $N_{2}^{-}$is irrelevant with corresponding $h$ becoming positive for $q_{1}>q_{1}^{\text {th }}$ (threshold value).

The deformed contour of integration can be chosen such that it passes through all the relevant saddle points (depicted in blue-triangle and blue-square), and follow closely the Lefschetz-thimbles passing though them. The saddles depicted by blue-triangle is predominantly imaginary, and hence correspond to a predominantly Euclidean geometry, while the saddle point depicted in blue-square is predominantly real and hence correspond to a predominantly Lorentzian geometry.

The deformed contour starts at blue-triangle then circles around following the Lefschetz-thimble (red-line) lying in lower-right quadrant. Then it approaches origin. Thereafter near the origin it turns back, hovers around in the green region following the steepest-descent line approaching the blue-square. Thereafter it follows the Lefschetzthimble (red line) connecting blue-square in the upper-right quadrant. For the two relevant saddle the corresponding $\dot{q}_{0}$ at the initial boundary can be computed using eq. (6.2). As the $N_{c}$ at the two saddle point is different, as a result the initial $\dot{q}_{0}$ is different, indicating that the final geometry is a super-position of two different initial configurations.

We numerically compute the transition amplitude in this particular case and plot it in figure 4 as a function of $q_{1}$. For this situation under consideration we have $\theta_{\mathbf{\Delta}}=0$ and $\theta$ - $=\pi / 4$. Both saddle-points contribute in exponentially suppressed manner as $h<0$ for both of them. But this suppression varies with respect to $q_{1}$. At each $q_{1}$ the weight of blue-square saddle point is more than the weight of blue-triangle saddle favouring a Lorentzian geometry. 


\section{Complex initial momentum}

In this section we consider a very simple model of no-boundary proposal where we directly fix the initial field derivative $\dot{q}_{0}$. Certainly, this scenario fall in case (b) category as discussed in subsection 3.3. To properly motivate the choice of $\dot{q}_{0}$ we start by considering deSitter (dS) geometry which will be solution to bulk equation of motion. This means that for $\Lambda=3 \lambda^{2}>0$ in $d=4$ we have for the spacetime dS metric in eq. (1.2)

$$
N_{p}=1, \quad a\left(t_{p}\right)=\frac{1}{\lambda} \cosh \left(\lambda t_{p}\right) .
$$

$\mathrm{dS}$ can be embedded in 5-dimensions where in closed slicing it can pictured as hyperboloid having a minimum spatial extent at $t_{p}=0$. The intuition behind the no-boundary proposal is that the geometry is rounded off, so as to have no boundary in the beginning of time. This can be achieved by analytically continuing the original dS metric to Euclidean time, starting exactly at the waist of hyperboloid at $t_{p}=0$. This means

$$
t_{p}=\mp i\left(\tau-\frac{\pi}{2 \lambda}\right), \quad 0 \leq \tau \leq \frac{\pi}{2 \lambda} .
$$

This means that along the Euclidean section the dS metric transforms in to that of a 4-sphere

$$
\mathrm{d} s^{2}=\mathrm{d} \tau^{2}+\frac{1}{\lambda^{2}} \sin ^{2}(\lambda \tau) \mathrm{d} \Omega_{3}^{2} .
$$

This geometry has no boundary at $\tau=0$ and smoothly closes off.

It should be emphasised that there are two possibilities of the time rotation to Euclidean time above, corresponding to the sign appearing in eq. (7.2). Each of these choices correspond to a different Wick rotation. The upper sign correspond to the standard Wick rotation which is also used in the flat spacetime QFT. It is also the sign chosen in the work of Hartle and Hawking $[37,59]$. For this sign the perturbations around the geometry are stable and suppressed. The lower sign in eq. (7.2) correspond to Vilenkin's tunneling geometry where small perturbation around the geometry are unsuppressed [39, 47]. The process of Wick rotation can also be thought of the lapse $N_{p}$ changing its value from $N_{p}=1$ to $N_{p}=\mp i$, thereby implying that the total time $T_{p}=\int N_{p} \mathrm{~d} t_{p}$ becoming complex valued.

This can be translated into the language of metric in eq. (2.11) and will thereby imply

$$
\sinh \left(\lambda t_{p}\right)=\lambda^{2} N_{\mathrm{HH}} t+i,
$$

where $N_{\mathrm{HH}}$ will turn out to the saddle-point value of the lapse integral corresponding to Hartle-Hawking geometry [37, 59]. It is given by

$$
N_{\mathrm{HH}}=\frac{\sqrt{\lambda^{2} q_{1}-1}}{\lambda^{2}}-\frac{i}{\lambda^{2}}
$$

where $q_{1}=q(t=1)$. The HH-geometry fall in case $(b)$ of the mixed boundary conditions. On comparing it with eq. (3.23) one has $q(t=0)=0$, while the $q(t)$ is given by

$$
\dot{q}_{0}=q_{1}-\lambda^{2} N_{\mathrm{HH}}^{2}, \quad \Rightarrow \quad q_{\mathrm{HH}}(t)=\lambda^{2} N_{\mathrm{HH}}^{2} t^{2}+\left(q_{1}-\lambda^{2} N_{\mathrm{HH}}^{2}\right) t
$$


where $0 \leq t \leq 1$. From this we can immediately notice the complex nature of saddle point value $N_{\mathrm{HH}}$ encodes the direction of Wick rotation. This can be seen by computing the momentum using eq. (3.21) at $t=0$

$$
\left.\frac{\dot{q}_{H H}}{2 N_{\mathrm{HH}}}\right|_{t=0}=+i
$$

Motivated by the Hartle-Hawking geometry [37, 59] where we notice that the initial momentum is complex and appears with positive sign resulting in a stable and suppressed behaviour of fluctuations, we can consider appling this boundary condition in the case $(b)$ scenario that is considered in this paper. More clearly motivated by the works of HartleHawking $[37,59]$, we choose the following mixed boundary condition in the case $(b)$

$$
\dot{q}_{0}^{(b)}=+2 i N_{c}, \quad q^{(b)}(t=1)=q_{1} .
$$

Plugging this special condition in eq. (3.23) and (3.25) we get

$$
q^{(b)}(t)=\lambda^{2} N_{c}^{2} t^{2}+2 i N_{c} t+q_{1}-2 i N_{c}-\lambda^{2} N_{c}^{2},
$$

and the corresponding action for lapse $N_{c}$ is given by

$$
S_{\mathrm{tot}}^{\mathrm{HH}}=\frac{V_{3}}{16 \pi G}\left[2 \lambda^{4} N_{c}^{3}+6 i \lambda^{2} N_{c}^{2}-6 q_{1} \lambda^{2} N_{c}-2 i\left\{3 q_{1}+8 \alpha\right\}\right]
$$

respectively. There are few crucial things to note here for this special mixed boundary condition: (1) the action for lapse $N_{c}$ is complex (2) the action is no longer singular at $N_{c}=0$. The former is a direct consequence of the imposition of complex initial momentum which subsequently leads to complex geometries. A complex action will mean that even for real values of lapse $N_{c}$ there will be a non-zero weighting of the corresponding geometrical configuration.

The later point about the lack of $N_{c}=0$ singularity can be understood by realising that as we are fixing the initial momentum (and not the initial size of geometry). As result we are summing over all possible initial 3-geometry size and their transition to 3-geometry of size $q_{1}$. This will also include a transition from $q_{1} \rightarrow q_{1}$. Such a transition can occur instantaneously i.e. with $N_{c}=0$. This means that there is nothing singular happening at $N_{c}=0$.

An interesting third observation is that the saddle point equation following from action in eq. (7.10) is quadratic in $N_{c}$.

$$
\frac{\mathrm{d} S_{\mathrm{tot}}^{\mathrm{HH}}}{\mathrm{d} N_{c}}=0 \quad \Rightarrow \quad \lambda^{4} N_{c}^{2}+2 i \lambda^{2} N_{c}-q_{1} \lambda^{2}=0
$$

This quadratic equation has only two saddle point solution, unlike the scenarios studied in previous section where there were six saddle points. In the present case the saddle points are also independent of the Gauss-Bonnet coupling $\alpha$. These saddle points have a very simple expression

$$
N_{ \pm}=\frac{-i \pm \sqrt{q_{1} \lambda^{2}-1}}{\lambda^{2}}
$$


It should be noted that $N_{+}$is same as the saddle point considered in the work of HartleHawking [37, 59].

At this point our interest is to compute eq. (4.8) for the case (b) for the boundary condition mentioned in eq. (7.8). The $N_{c^{-}}$action is given in eq. (7.10). As the integrand is not singular for $N_{c}=0$, so one can extend the range of the $N_{c}$-integration from $-\infty$ to $\infty$. Then we have

$$
G\left[\dot{q}_{0}=2 i N_{c}, q_{1}\right]=\frac{1}{2 \sqrt{\pi i}} \int_{-\infty}^{\infty} \mathrm{d} N_{c} \exp \left(\frac{i}{\hbar} S_{\mathrm{tot}}^{\mathrm{HH}}\right)
$$

This can be performed using the Picard-Lefschetz and WKB methods. Once the saddle points for the action $S_{\text {tot }}^{\mathrm{HH}}$ are known, one can compute the steepest ascent/descent flow lines corresponding to each of the saddle point. A saddle point is termed relevant if the steepest ascent path emanating from it hits the original integration contour which is $(-\infty,+\infty)$. If the action is real then it implies that the relevant saddle points will have their corresponding Morse-function $h<0$. However, in the case when action is complex this obstruction can be evaded.

The analyse the nature of Morse-function at each saddle point we first compute the on-shell action, which is obtained by plugging the saddle point solution given in eq. (7.12) back in the action given in eq. (7.10). The on-shell action at the two saddle points is given by,

$$
S_{ \pm}^{\mathrm{HH}}=2 \pi^{2}\left[-i\left(\frac{1}{\lambda^{2}}+4 \alpha\right) \mp \frac{\left(q_{1} \lambda^{2}-1\right)^{3 / 2}}{\lambda^{2}}\right]
$$

It should be emphasised here that only the imaginary part of the action gets correction from the Gauss-Bonnet sector of gravity while the real parts remains unaffected and is same as for pure Einstein-Hilbert gravity. This immediately implies that for $q_{1}>1 / \lambda^{2}$, the Morse-function for the two saddle points is

$$
h\left(N_{ \pm}\right)=\frac{2 \pi^{2}}{\hbar}\left(\frac{1}{\lambda^{2}}+4 \alpha\right) .
$$

It is real-positive and independent of $q_{1}$. However, it receives a correction from the GaussBonnet sector of gravity action. By analysing the steepest ascent flow lines emanating from both the saddle points it is realised that both of them are relevant. Even though for both of them $h\left(N_{ \pm}\right)>0$.

In figure 5 we plot the various flow-line, saddle points, forbidden/allowed regions. As both the saddle points are relevant, so the corresponding Lefschetz thimbles passing through both the saddle points constitute the deformed contour of integration. This deformed contour starts at upper-left quadrant, follow the red-line, crosses the negative real-axis then goes over to lower-left quadrant and asymptotes to $-i \infty$. The second part of contour starts $-i \infty$ in lower-right quadrant, follows the red-line, crosses the positive realaxis, then goes to the upper-right quadrant following the red-line. The Picard-Lefschetz 


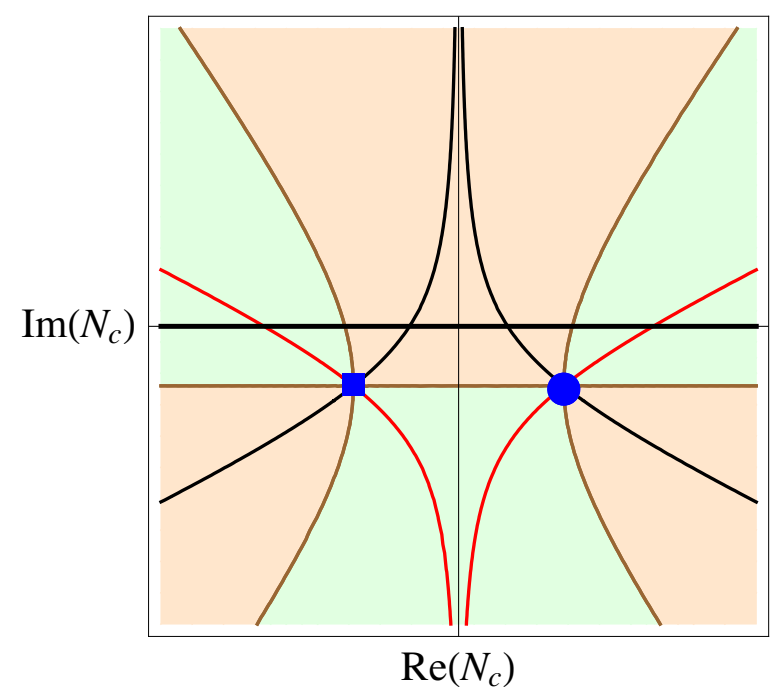

Figure 5. We consider the case of no-boundary Universe where we impose the mixed boundary condition: with Euclidean momentum at $t=0$ and fixed final size at $t=1$. The lapse action given in eq. (7.11) is complex. We take $\dot{q}_{0}=+2 i N_{c}$ motivated by work of Hartle-Hawking [37, 59]. For the purpose of this numerical example we take $\lambda=1, \alpha=2$. We choose final boundary condition to be $q_{1}=3$. We plot on $x$-axis real-part of $N_{c}$ while the $y$-axis is imaginary part of $N_{c}$. The red lines correspond to steepest descent lines (thimbles $\mathcal{J}_{\sigma}$ ), while the thin black lines are steepest ascent lines and denoted by $\mathcal{K}_{\sigma}$. Both the saddle points are depicted in blue: $N_{-}$(blue-square) and $N_{+}$ (blue-circle). Both saddle points are relevant. The steepest ascent lines emanating from both of them intersects the original integration contour $(-\infty,+\infty)$ which is shown by thick-black line. The Morse-function $h$ is same for both saddle points: $h\left(N_{ \pm}\right)>0 . H$ remains constant along the red and thin-black lines, and is equal to the value of $H\left(N_{\sigma}\right)$. The light-green region is allowed region with $h<h\left(N_{\sigma}\right)$ for all values of $\sigma$. The light-orange region (forbidden region) has $h>h\left(N_{\sigma}\right)$ for all $\sigma$. The boundary of these region is depicted in brown lines. Along these line we have $h=h\left(N_{\sigma}\right)$.

theory then gives the transition amplitude in the saddle point approximation as

$$
\begin{aligned}
G\left[\dot{q}_{0}=2 i N_{c}, q_{1}\right] & =\frac{1}{2 \sqrt{\pi i}}\left[\exp \left(\frac{i S_{\mathrm{tot}}^{\mathrm{HH}}\left(N_{-}\right)}{\hbar}\right)+\exp \left(\frac{i S_{\mathrm{tot}}^{\mathrm{HH}}\left(N_{+}\right)}{\hbar}\right)\right] \\
& =\frac{e^{-i \pi / 4}}{\sqrt{\pi}} \exp \left[\frac{2 \pi^{2}}{\hbar}\left(\frac{1}{\lambda^{2}}+4 \alpha\right)\right] \cos \left[\frac{2 \pi^{2} \lambda}{\hbar}\left(q_{1}-\frac{1}{\lambda^{2}}\right)^{3 / 2}\right] .
\end{aligned}
$$

We notice that we get a non-perturbative correction from the Gauss-Bonnet sector of gravity to the Hartle-Hawking wave-function from a Lorentzian path-integral. This transitional amplitude is fully non-perturbative and incorporates the non-trivial features coming from the Gauss-Bonnet coupling.

\section{Conclusion}

In this paper we study the path-integral of gravitational theory where the gravitational dynamics is governed by Einstein-Hilbert gravity with an addition of Gauss-Bonnet gravity. We study this setup in four spacetime dimensions directly in Lorentzian signature. In four 
spacetime dimensions the Gauss-Bonnet sector of gravity action is also topological and doesn't contribute in the bulk dynamics. However it has a crucial role to play at boundaries. Depending on the nature of boundary conditions the Gauss-Bonnet modifications will affect the study of path-integral. This paper aims to investigate these issues by considering the gravitational path-integral in a reduced setup of mini-superspace approximation.

We start by considering the mini-superspace action of the theory and vary it with respect to field variables to study the dynamical equation of motion and the nature of boundary terms. To have a consistent boundary value problem one has to incorporate additional terms at the boundary. We notice that with Neuman boundary condition one ends up with inconsistencies in fixing the free parameters in the solution to the equation of motion. With Dirichlet boundary conditions on other hand it is seen that no nontrivial effects arise from the Gauss-Bonnet sector. However, in the case of mixed boundary conditions (where one specifies $q(t)$ at one end point and its derivative $\dot{q}(t)$ at another end point) we notice that the Gauss-Bonnet sector starts to play a non-trivial role. Although the solution to the equation of motion for $q(t)$ doesn't get contribution from the Gauss-Bonnet sector, however, the action for the lapse $N_{c}$ gets non-trivial addition due the non-vanishing boundary terms. Such non-trivialities arising from the Gauss-Bonnet sector later leads to richer features while evaluating the integration over lapse $N_{c}$ in eq. (4.1).

The paper aims to study the transition amplitude from one 3-geometry to another and investigate the circumstances under which the Gauss-Bonnet sector starts to affects this amplitude in a non-trivial manner. Such a transition amplitude is dictated by a pathintegral over $q(t)$ and a contour integration over lapse $N_{c}$. The path-integral over $q(t)$ can be performed exactly as the Gauss-Bonnet part controls only the boundary, while the bulk remains unaffected. The path-integral over $q(t)$ is governed entirely by the Einstein-Hilbert part of gravity action. Once the integral over $q(t)$ respecting the boundary conditions is performed, we are left with an contour integration over lapse $N_{c}$ given in eq. (4.8), with the integrand containing non-trivial features coming from the Gauss-Bonnet sector.

We analyse this contour integration by lifting lapse $N_{c}$ to complex plane and making use of Picard-Lefschetz theory to investigate the nature of integrand. We find the saddle points of the $N_{c}$-action and realise that they occur in three pairs. This is a new feature of the Gauss-Bonnet gravity which is absent in the case of pure Einstein-Hilbert gravity having only two (or less) pairs of saddle points. In the mixed boundary conditions case the Gauss-Bonnet sector contributes non-trivially and give rise to additional saddle points in the complex $N_{c}$ plane. The three pairs of saddle points follow from the cubic saddle-point equation in $N_{c}^{2}$. Moreover, if the cubic polynomial equation has real coefficients then the nature of saddle points can be determined by analysing the discriminant $\Delta$ of the cubic equation, which in turn depend on parameter values and boundary conditions.

As an application of this we considered an example of no-boundary Universe, and analyse the transition amplitude in this setup. In this situation the initial $q_{0}=0$. This has consequences: in case $(a) \dot{q}_{1}^{(a)}$ and $q_{1}^{(a)}$ are related, while in case $(b) \dot{q}_{0}^{(b)}$ and $q_{1}^{(b)}$ are related. In either case we have multiple relevant saddle points, so this implies that for realpositive $q_{1}$ in the case $(a)$ we will have multiple possibilities for $\dot{q}_{1}^{(a)}$. This is contradictory to our original boundary condition requirement where $\dot{q}_{1}^{(a)}$ is supposed to be fixed at the 
final boundary. Case $(a)$ is therefore ruled out. Such a contradiction doesn't happen in case $(b)$. In case $(b)$ for a fixed real-positive $q_{1}^{(b)}$ there are multiple value for $\dot{q}_{0}^{(b)}$ for the corresponding relevant saddle-points. This is acceptable as the final geometry can be seen as arising from the superposition of multiple allowed initial configurations.

In case (b) by making use of the relation in eq. (6.2) one can obtain the action for lapse $N_{c}$ entirely in terms of $q_{1}$. As $q_{1}$ is positive, so the action for lapse is entirely real. The saddle point equation is cubic in $N_{c}^{2}$ with real coefficients. We realise that for positive $k$, $\Lambda$, and $\alpha$ there are three distinct real roots for $N_{c}^{2}$ : one positive and two negative. This implies that one saddle point is always real-positive while its 'twin' is real-negative. There are four saddle points lying on imaginary axis: two on positive imaginary axis while their conjugate twins on negative imaginary axis. There are total of six saddle-points, which is new compared to the pure Einstein-Hilbert gravity. Attached to each saddle-point there are two Lefschetz thimbles and two steepest ascent lines. Only three of the saddle point can be reached by flowing downward along the steepest ascent lines starting from the original integration contour $\left(0^{+}, \infty\right)$. Out of the three only two have their corresponding Morsefunction $h<0$, making them relevant. One of the relevant saddle-point lies on negative imaginary axis while other lies on positive real-axis. The deformed contour of integration passes through these relevant saddles following the Lefschetz-thimbles. The deformed contour therefore incorporate contributions from both saddles resulting in interference. The full amplitude is a superposition of the contribution coming from two configurations with the more weight associated to Lorentzian saddle compared to Euclidean saddle.

We consider another special case of no-boundary proposal with a complex initial momentum. Here we are inspired by the past works of Hartle-Hawking [37, 59], where the authors noticed that a particular choice of Wick-rotation leads to stable and suppressed perturbations. This choice of Wick-rotation eventually implies that the initial momentum in the cosmic evolution was complex. Inspired by their work we choose a special initial boundary condition to be $\dot{q}_{0}^{(b)}=+2 i N_{c}$. This particular scenario falls in the category of case $(b)$ of mixed boundary conditions. We notice that in particular in this situation the lapse $N_{c}$ action is complex, and that the action is non-singular at $N_{c}=0$. Moreover, in this case we have only two saddle-points and both are relevant. We compute the transition amplitude from the initial to final configuration and obtain an analogue of Hartle-Hawking wave-function having a non-trivial and non-perturbative correction from the Gauss-Bonnet sector of gravity theory.

Certainly, more work needs to be done in this direction as many things are still unexplored. In the study in section 6 we haven't directly fixed the initial line $\dot{q}_{0}$ in the case (b), rather sort of derived it by imposing condition that $q_{0}^{(b)}=0$ and $q_{1}^{(b)}>0$. This two requirements eventually leads to two relevant saddle points. For each of these saddle point there is a corresponding fixed $\dot{q}_{0}^{(b)}$. This is hinting at fact that the final geometry of Universe is arising due to superposition of the two very different initial configurations. Perhaps there are two different copies of Universe initially whose evolution and interference resulted in the final geometry of the Universe. Were these two Universe entangled in past and overtime this entanglement grew stronger resulting in current Universe? This is hard to answer in present manuscript. 
Another crucial thing missing in this paper is an analysis about the behaviour of fluctuations, which are important to understand the stability of Universe. In past works on no-boundary Universe it was noticed that such models are unstable to fluctuations [39]. This is a worrisome feature which if it exists make the model less reliable. Past attempts to overcome these issues involved imposing different types of boundary conditions for background and for fluctuations $[49,50]$. It is worth asking this same question in the case of the Gauss-Bonnet gravity too. Does the Gauss-Bonnet modifications leads to a more stable behaviour of fluctuations? If not then what kind of boundary conditions should be imposed for the fluctuations? Moreover, in the case of HH-model the choice of Wick-rotation leads to stable and suppressed behaviour of fluctuations [37, 59]. Currently it is not clear whether these fluctuations will remain suppressed when non-perturbative corrections from Gauss-Bonnet gravity are incorporated. We plan to address this in our future work.

\section{Acknowledgments}

I will like to thank Jean-Luc Lehners, Alok Laddha, Nirmalya Kajuri and Avinash Raju for useful discussions. GN is supported by "Zhuoyue" (distinguished) Fellowship (ZYBH2018$03)$.

Open Access. This article is distributed under the terms of the Creative Commons Attribution License (CC-BY 4.0), which permits any use, distribution and reproduction in any medium, provided the original author(s) and source are credited.

\section{References}

[1] G. 't Hooft and M.J.G. Veltman, One loop divergencies in the theory of gravitation, Ann. Henri PoincareÁ 20 (1974) 69.

[2] S. Deser, H.-S. Tsao and P. van Nieuwenhuizen, Nonrenormalizability of Einstein Yang-Mills Interactions at the One Loop Level, Phys. Lett. B 50 (1974) 491 [INSPIRE].

[3] S. Deser and P. van Nieuwenhuizen, One Loop Divergences of Quantized Einstein-Maxwell Fields, Phys. Rev. D 10 (1974) 401 [inSPIRE].

[4] S. Deser and P. van Nieuwenhuizen, Nonrenormalizability of the Quantized Dirac-Einstein System, Phys. Rev. D 10 (1974) 411 [inSPIRE].

[5] M.H. Goroff and A. Sagnotti, Quantum gravity at two loops, Phys. Lett. B 160 (1985) 81 [INSPIRE].

[6] M.H. Goroff and A. Sagnotti, The Ultraviolet Behavior of Einstein Gravity, Nucl. Phys. B 266 (1986) 709 [INSPIRE].

[7] A.E.M. van de Ven, Two loop quantum gravity, Nucl. Phys. B 378 (1992) 309 [INSPIRE].

[8] K.S. Stelle, Renormalization of Higher Derivative Quantum Gravity, Phys. Rev. D 16 (1977) 953 [INSPIRE].

[9] A. Salam and J.A. Strathdee, Remarks on High-energy Stability and Renormalizability of Gravity Theory, Phys. Rev. D 18 (1978) 4480 [INSPIRE]. 
[10] J. Julve and M. Tonin, Quantum Gravity with Higher Derivative Terms, Nuovo Cim. B 46 (1978) 137 [INSPIRE].

[11] G. Narain and R. Anishetty, Short Distance Freedom of Quantum Gravity, Phys. Lett. B $\mathbf{7 1 1}$ (2012) 128 [arXiv:1109.3981] [INSPIRE].

[12] G. Narain and R. Anishetty, Unitary and Renormalizable Theory of Higher Derivative Gravity, J. Phys. Conf. Ser. 405 (2012) 012024 [arXiv: 1210.0513] [INSPIRE].

[13] G. Narain, Signs and Stability in Higher-Derivative Gravity, Int. J. Mod. Phys. A 33 (2018) 1850031 [arXiv:1704.05031] [INSPIRE].

[14] G. Narain, Exorcising Ghosts in Induced Gravity, Eur. Phys. J. C 77 (2017) 683 [arXiv: 1612.04930] [INSPIRE].

[15] A. Codello and R. Percacci, Fixed points of higher derivative gravity, Phys. Rev. Lett. 97 (2006) 221301 [hep-th/0607128] [INSPIRE].

[16] M.R. Niedermaier, Gravitational Fixed Points from Perturbation Theory, Phys. Rev. Lett. 103 (2009) 101303 [INSPIRE].

[17] A. Salvio and A. Strumia, Agravity, JHEP 06 (2014) 080 [arXiv:1403.4226] [INSPIRE].

[18] D. Lovelock, The Einstein tensor and its generalizations, J. Math. Phys. 12 (1971) 498 [INSPIRE].

[19] D. Lovelock, The four-dimensionality of space and the Einstein tensor, J. Math. Phys. 13 (1972) 874 [INSPIRE].

[20] C. Lanczos, A Remarkable property of the Riemann-Christoffel tensor in four dimensions, Annals Math. 39 (1938) 842 [INSPIRE].

[21] J. York, Boundary terms in the action principles of general relativity, Found. Phys. 16 (1986) 249 [INSPIRE].

[22] J.D. Brown and J.W. York, Jr., The Microcanonical functional integral. 1. The Gravitational field, Phys. Rev. D 47 (1993) 1420 [gr-qc/9209014] [INSPIRE].

[23] C. Krishnan and A. Raju, A Neumann Boundary Term for Gravity, Mod. Phys. Lett. A 32 (2017) 1750077 [arXiv: 1605.01603] [InSPIRE].

[24] E. Witten, A Note On Boundary Conditions In Euclidean Gravity, arXiv:1805.11559 [INSPIRE].

[25] C. Krishnan, S. Maheshwari and P.N. Bala Subramanian, Robin Gravity, J. Phys. Conf. Ser. 883 (2017) 012011 [arXiv:1702.01429] [INSPIRE].

[26] I.A. Batalin and G.A. Vilkovisky, Relativistic S Matrix of Dynamical Systems with Boson and Fermion Constraints, Phys. Lett. B 69 (1977) 309 [INSPIRE].

[27] C. Teitelboim, Quantum Mechanics of the Gravitational Field, Phys. Rev. D 25 (1982) 3159 [INSPIRE].

[28] C. Teitelboim, The Proper Time Gauge in Quantum Theory of Gravitation, Phys. Rev. D 28 (1983) 297 [InSPIRE].

[29] J.J. Halliwell, Derivation of the Wheeler-De Witt Equation from a Path Integral for Minisuperspace Models, Phys. Rev. D 38 (1988) 2468 [InSPIRE].

[30] C. Teitelboim, Causality Versus Gauge Invariance in Quantum Gravity and Supergravity, Phys. Rev. Lett. 50 (1983) 705 [INSPIRE]. 
[31] G.W. Gibbons, S.W. Hawking and M.J. Perry, Path Integrals and the Indefiniteness of the Gravitational Action, Nucl. Phys. B 138 (1978) 141 [InSPIRE].

[32] P. Candelas and D.J. Raine, Feynman Propagator in Curved Space-Time, Phys. Rev. D 15 (1977) 1494 [InSPIRE].

[33] M. Visser, How to Wick rotate generic curved spacetime, arXiv:1702.05572 [INSPIRE].

[34] A. Baldazzi, R. Percacci and V. Skrinjar, Quantum fields without Wick rotation, Symmetry 11 (2019) 373 [arXiv: 1901.01891] [INSPIRE].

[35] A. Baldazzi, R. Percacci and V. Skrinjar, Wicked metrics, Class. Quant. Grav. 36 (2019) 105008 [arXiv:1811.03369] [INSPIRE].

[36] S.W. Hawking, The Boundary Conditions of the Universe, Pontif. Acad. Sci. Scr. Varia 48 (1982) 563, PRINT-82-0179, Cambridge U.K. (1982).

[37] J.B. Hartle and S.W. Hawking, Wave Function of the Universe, Adv. Ser. Astrophys. Cosmol. 3 (1987) 174 [Phys. Rev. D 28 (1983) 2960] [INSPIRE].

[38] J. Feldbrugge, J.-L. Lehners and N. Turok, Lorentzian Quantum Cosmology, Phys. Rev. D 95 (2017) 103508 [arXiv:1703.02076] [INSPIRE].

[39] J. Feldbrugge, J.-L. Lehners and N. Turok, No smooth beginning for spacetime, Phys. Rev. Lett. 119 (2017) 171301 [arXiv:1705.00192] [INSPIRE].

[40] J. Feldbrugge, J.-L. Lehners and N. Turok, No rescue for the no boundary proposal: Pointers to the future of quantum cosmology, Phys. Rev. D 97 (2018) 023509 [arXiv:1708.05104] [INSPIRE].

[41] A. Vilenkin, Creation of Universes from Nothing, Phys. Lett. B 117 (1982) 25 [InSPIRE].

[42] A. Vilenkin, The Birth of Inflationary Universes, Phys. Rev. D 27 (1983) 2848 [INSPIRE].

[43] A. Vilenkin, Quantum Creation of Universes, Phys. Rev. D 30 (1984) 509 [InSPIRE].

[44] S.W. Hawking, The Quantum State of the Universe, Adv. Ser. Astrophys. Cosmol. 3 (1987) 236 [Nucl. Phys. B 239 (1984) 257] [INSPIRE].

[45] G.W. Gibbons, The Einstein Action of Riemannian Metrics and Its Relation to Quantum Gravity and Thermodynamics, Phys. Lett. A 61 (1977) 3 [INSPIRE].

[46] J.J. Halliwell and J. Louko, Steepest Descent Contours in the Path Integral Approach to Quantum Cosmology. 1. The de Sitter Minisuperspace Model, Phys. Rev. D 39 (1989) 2206 [INSPIRE].

[47] J.J. Halliwell and J.B. Hartle, Integration Contours for the No Boundary Wave Function of the Universe, Phys. Rev. D 41 (1990) 1815 [INSPIRE].

[48] J.J. Halliwell and J.B. Hartle, Wave functions constructed from an invariant sum over histories satisfy constraints, Phys. Rev. D 43 (1991) 1170 [INSPIRE].

[49] A. Di Tucci and J.-L. Lehners, No-Boundary Proposal as a Path Integral with Robin Boundary Conditions, Phys. Rev. Lett. 122 (2019) 201302 [arXiv:1903.06757] [INSPIRE].

[50] A. Di Tucci, J.-L. Lehners and L. Sberna, No-boundary prescriptions in Lorentzian quantum cosmology, Phys. Rev. D 100 (2019) 123543 [arXiv:1911.06701] [INSPIRE].

[51] N. Deruelle and L. Farina-Busto, The Lovelock Gravitational Field Equations in Cosmology, Phys. Rev. D 41 (1990) 3696 [INSPIRE]. 
[52] F.R. Tangherlini, Schwarzschild field in $n$ dimensions and the dimensionality of space problem, Nuovo Cim. 27 (1963) 636 [INSPIRE].

[53] F. Tangherlini, Dimensionality of Space and the Pulsating Universe, Nuovo Cim. B 91 (1986) 209.

[54] A. Di Tucci, M.P. Heller and J.-L. Lehners, Lessons for quantum cosmology from anti-de Sitter black holes, Phys. Rev. D 102 (2020) 086011 [arXiv:2007.04872] [InSPIRE].

[55] E. Witten, Analytic Continuation Of Chern-Simons Theory, AMS/IP Stud. Adv. Math. 50 (2011) 347 [arXiv: 1001.2933] [INSPIRE].

[56] E. Witten, A New Look At The Path Integral Of Quantum Mechanics, arXiv:1009.6032 [INSPIRE].

[57] G. Basar, G.V. Dunne and M. Ünsal, Resurgence theory, ghost-instantons, and analytic continuation of path integrals, JHEP 10 (2013) 041 [arXiv: 1308.1108] [INSPIRE].

[58] Y. Tanizaki and T. Koike, Real-time Feynman path integral with Picard-Lefschetz theory and its applications to quantum tunneling, Annals Phys. 351 (2014) 250 [arXiv:1406.2386] [INSPIRE].

[59] J.J. Halliwell and S.W. Hawking, The Origin of Structure in the Universe, Adv. Ser. Astrophys. Cosmol. 3 (1987) 277 [Phys. Rev. D 31 (1985) 1777] [INSPIRE]. 